Report no. 98/14

\title{
Stabilized hp-Finite Element Methods for First-Order Hyperbolic Problems
}

\author{
Paul Houston \\ Oxford University Computing Laboratory, Wolfson Building, \\ Parks Road, Oxford OX1 3QD, UK \\ Ch. Schwab \\ Seminar of Applied Mathematics, ETH Zürich, \\ CH-8092 Zürich, Switzerland \\ Endre Süli \\ Oxford University Computing Laboratory, Wolfson Building, \\ Parks Road, Oxford OX1 3QD, UK
}

We analyze the $h p$-version of the streamline-diffusion (SDFEM) and of the discontinuous Galerkin method (DGFEM) for first-order linear hyperbolic problems. For both methods, we derive new error estimates on quadrilateral meshes which are sharp in the mesh-width $h$ and in the spectral order $p$ of the method, assuming that the stabilization parameter is $O(h / p)$. For piecewise analytic solutions, exponential convergence is established. For the DGFEM we admit very general irregular meshes and for the SDFEM we allow meshes which contain hanging nodes. Numerical experiments confirm the theoretical results.

Oxford University Computing Laboratory

Numerical Analysis Group

Wolfson Building

Parks Road

Oxford, England OX13QD

November, 1998 


\section{Introduction}

The numerical solution of first-order hyperbolic problems by finite element methods has become increasingly popular in recent years. Two major families of methods have emerged: the streamline diffusion method (SDFEM) and the discontinuous Galerkin method (DGFEM). While the SDFEM uses continuous approximations, the DGFEM allows for discontinuities at element interfaces and is, in spirit, close to the well-established finite volume schemes with some particular differences, however.

For classical finite element and finite volume methods improvement in accuracy relies on mesh refinement while keeping the approximation order within the elements (or cells) at a fixed, low value, leading to the so-called $h$-version convergence. In the late seventies and early eighties, however, the so-called $p$-version or spectral methods emerged which achieve convergence by increasing the polynomial order of the approximation rather than by mesh refinement. Naturally, this is very advantageous in situations where a smooth or even analytic solution is to be approximated. Unfortunately, the solution to most problems of practical interest is only piecewise analytic: in elliptic problems (such as stationary viscous incompressible flow), corner and edge singularities arise in the vicinity of which the solution regularity is very low. Good performance of high order methods and, in particular, spectral or exponential convergence for such problems mandates the combination of increasing polynomial degree in regions where the solution is smooth and mesh refinement with low order polynomial approximations close to singularities. This strategy gives rise to the $h p$-version of the finite element method introduced by Babuška and his co-workers in the mid-eighties for elliptic problems.

The DGFEM has been proposed and first analyzed in [9] for a linear hyperbolic problem. There, the method was formulated and its $h$-version convergence was established in $L^{2}(\Omega)$, albeit with a suboptimal rate. Later, in [7], [8], the optimal rate of $O\left(h^{p+1 / 2}\right)$ in a meshdependent norm (stronger than $L^{2}(\Omega)$ ) was proved, assuming that the finite element space consisted of piecewise polynomials of degree $p$. In the meantime, the DGFEM has also been successfully applied to nonlinear hyperbolic conservation laws (see, e.g., [4]).

The $h p$-version of the DGFEM has been introduced by K. Bey and J.T. Oden, who gave a-priori and a-posteriori error bounds in [1]. Their analysis produced error estimates which, for a fixed $p$ and as $h \rightarrow 0$, reduced to the optimal order estimates of [7], [8], but also indicated convergence as $p \rightarrow \infty$ for fixed $h>0$. These results were derived under the assumption that the stabilization parameter in element $K$ is of size $h_{K} / p_{K}^{2}$; however, the rate of this spectral convergence was suboptimal.

In the present paper, we generalize the results of [1] in several directions. We establish a unified framework for the $h p$-error analysis of the SDFEM and the stabilized DGFEM; on quadrilateral meshes we derive error estimates which are sharp both as $h \rightarrow 0$ and as $p \rightarrow \infty$. These optimal error bounds are derived assuming that the stabilization parameter for both the DGFEM and the SDFEM, and for the $h-, p$ - and the $h p$-version is $O\left(h_{K} / p_{K}\right)$, independent of the solution regularity. For the DGFEM we admit very general, irregular meshes and for the SDFEM we allow meshes which contain hanging nodes. Most importantly, our error estimates depend explicitly on the elemental solution regularity and indeed allow us to deduce exponential convergence rates for piecewise analytic solutions. The theoretical findings are in full agreement with the numerical experiments which complete the 
paper.

We note in closing that Bey and Oden [1] also considered the a-posteriori error analysis of the $h p$-DGFEM. Using the sharp error estimates obtained here, new a-posteriori error estimates can be derived for $h p$-DGFEM and $h p$-SDFEM. However, this subject is beyond the scope of the present paper and will be considered elsewhere.

\section{The model problem}

Let $\Omega$ be a bounded curved polyhedral domain in $\mathbb{R}^{d}, d \geq 2$. Given that $a=\left(a_{1}, \ldots, a_{d}\right)$ is a $d$-component vector function defined on $\bar{\Omega}$ with $a_{i} \in C^{1}(\bar{\Omega}), i=1, \ldots, d$, we define the following subsets of $\Gamma=\partial \Omega$ :

$$
\begin{aligned}
& \Gamma_{-}=\{x \in \Gamma: a(x) \cdot n(x)<0\}, \\
& \Gamma_{+}=\{x \in \Gamma: a(x) \cdot n(x)>0\},
\end{aligned}
$$

where $n(x)$ denotes the unit outward normal vector to $\Gamma$ at $x \in \Gamma$. It is assumed here implicitly that in these definitions $x$ ranges only through those points of $\Gamma$ at which $n(x)$ is defined; consequently, $\Gamma_{-}$and $\Gamma_{+}$are not necessarily connected subsets of $\Gamma$. For the sake of simplicity, we shall suppose that $\Gamma$ is non-characteristic in the sense that $\bar{\Gamma}_{-} \cup \bar{\Gamma}_{+}=\Gamma$.

Let $b \in C(\bar{\Omega}), f \in L_{2}(\Omega), g \in L_{2}\left(\Gamma_{-}\right)$and consider the hyperbolic boundary value problem

$$
\left\{\begin{aligned}
\mathcal{L} u \equiv a \cdot \nabla u+b u & =f \text { in } \Omega, \\
u & =g \text { on } \Gamma_{-} .
\end{aligned}\right.
$$

This problem has a unique weak solution $u \in L_{2}(\Omega)$ with $a \cdot \nabla u \in L_{2}(\Omega)$ and the boundary condition is satisfied as an equality in $L_{2}\left(\Gamma_{-}\right)$.

In the next two subsections we shall formulate the $h p$-streamline diffusion and $h p$ discontinuous finite element approximation of (2.1).

\section{$2.1 \quad h p$-Finite Element Spaces}

\subsubsection{Meshes}

Let $\mathcal{P}$ denote a partition of $\Omega$ into open patches $P$ which are images of a reference domain $\hat{P}$ under smooth, bijective maps $F_{P}$ :

$$
\forall P \in \mathcal{P}: \quad P=F_{P}(\hat{P}) .
$$

We assume that $\hat{P}$ is either the canonical cube

$$
\hat{P}=\hat{Q}:=(-1,1)^{d}
$$

or the unit simplex

$$
\hat{P}=\hat{S}:=\left\{\hat{x} \in \mathbb{R}^{d}: \hat{x}_{i}>0, \sum_{i=1}^{d} \hat{x}_{i}<1\right\}
$$




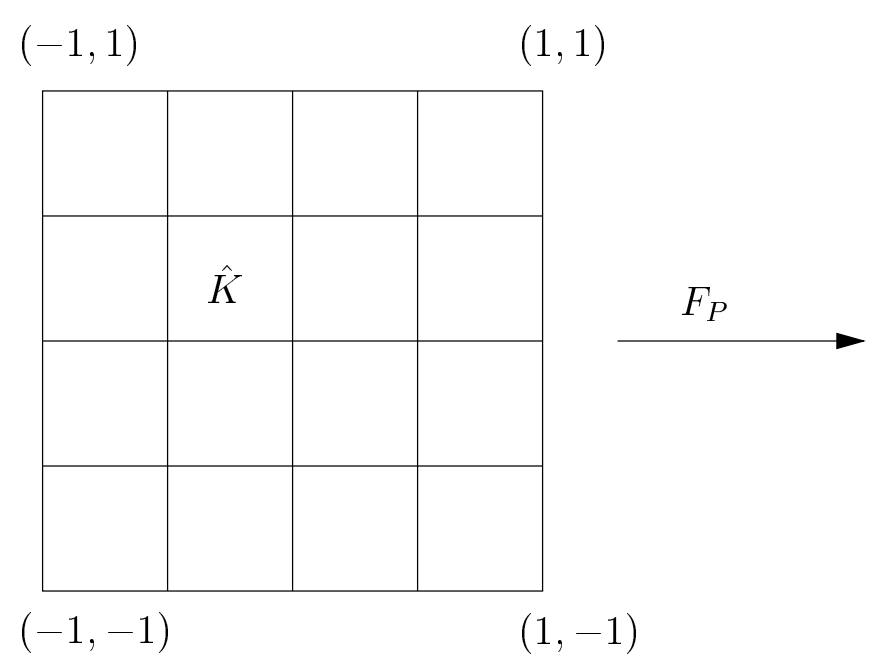

Patch $\hat{P}$ and Mesh $\hat{\mathcal{T}}_{P}$

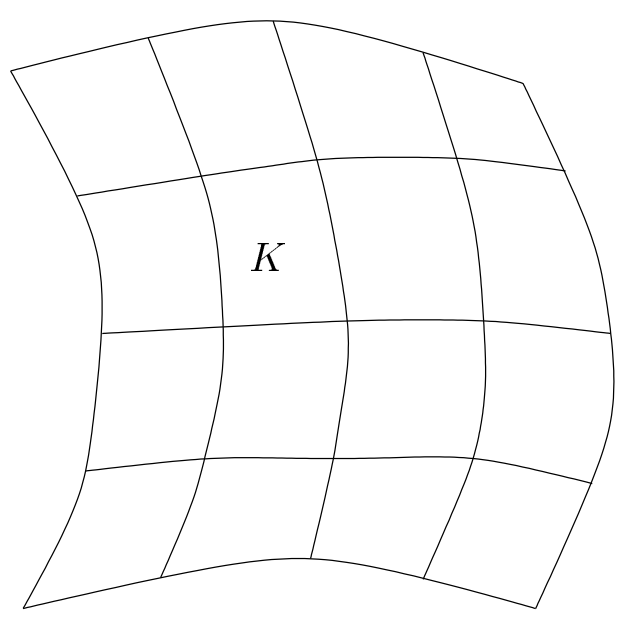

Patch $P$ and Mesh $\mathcal{T}_{P}$

Figure 1: Construction of the mesh patch $\mathcal{T}_{P}$ in the case when $\hat{P}$ is the canonical cube $\hat{Q}$.

At this stage, we consider meshes which result from either $\hat{Q}$ or $\hat{S}$; in Section 3 and onwards, for the sake of simplicity, we shall restrict ourselves to the case of $d=2$ and quadrilateral meshes.

The meshes $\mathcal{T}$ are constructed by subdividing the patches. For each $P$, a mesh $\mathcal{T}_{P}$ is obtained by first subdividing $\hat{P}$ into elements (e.g. triangles resp. quadrilaterals when $d=2$ ) labelled $\hat{K}$ which are affine equivalent to either $\hat{Q}$ or $\hat{S}$; we call this mesh $\hat{\mathcal{T}}_{P}$. A mesh $\mathcal{T}_{P}$ for $P \in \mathcal{P}$ is then obtained by simply mapping $\hat{\mathcal{T}}_{P}$ to $P$ using $F_{P}$ :

$$
\forall P \in \mathcal{P}: \mathcal{T}_{P}:=\left\{K \mid K=F_{P}(\hat{K}), \hat{K} \in \hat{\mathcal{T}}_{P}\right\}
$$

cf. Figure 1. As usual, the mesh $\mathcal{T}$ in $\Omega$ is the collection of all elements, i.e.

$$
\mathcal{T}=\bigcup_{P \in \mathcal{P}} \mathcal{T}_{P}
$$

Note that each $K \in \mathcal{T}$ is an image of the reference domain $\hat{P}$ via the element map $F_{K}$ : if $K \in P$ for some $P \in \mathcal{P}$,

$$
K=F_{K}(\hat{P}), \quad F_{K}:=F_{P} \circ A_{\hat{K}}
$$

and $A_{\hat{K}}: \hat{P} \rightarrow \hat{K} \in \hat{\mathcal{T}}_{P}$ is affine.

Remark 2.1 The maps $F_{P}, P \in \mathcal{P}$, are assumed to only deform the canonical patch $\hat{P}$ without any significant rescaling, thereby ensuring that the measure of the set $\hat{P}$ is comparable to the measure of set $P$; thus we may infer that the elements $\hat{K}$ in the mesh $\hat{\mathcal{T}}_{P}$ are of 
comparable size to the elements $K$ in the mesh $\mathcal{T}_{P}$. More explicitly, we assume there exist positive constants $c_{1}$ and $c_{2}$ such that for all $K$ in the mesh $\mathcal{T}$

$$
c_{1} \leq h_{K} / h_{\hat{K}} \leq c_{2}
$$

with $h_{K}=\operatorname{diam}(K), h_{\hat{K}}=\operatorname{diam}(\hat{K})$ and $\hat{K}$ is associated with $K$ via $K=F_{P}(\hat{K})$, as in (2.2). This will be important as our error estimates will be expressed in terms of Sobolev norms over the element domains $\hat{K}$, in order to ensure that only the scaling introduced by the affine element maps $A_{\hat{K}}$ is present in the analysis.

We emphasize that we could choose $A_{\hat{K}}$ and $F_{P}$ in (2.3) so as to obtain the usual parametric elements. However, it is also possible to use patches $P$ with structured patch-meshes $\mathcal{T}_{P}$, as e.g. geometric corner refinement, anisotropic boundary layer and edge refinement etc. In what follows, the partition $\mathcal{P}$ shall be fixed, i.e. mesh refinement is performed in $\hat{P}$.

We call the mesh $\mathcal{T}$ regular, if for any two $K, K^{\prime} \in \mathcal{T}$ the intersection $\bar{K} \cap \bar{K}^{\prime}$ is either empty or an entire boundary segment of dimension $0 \leq d^{\prime}<d$ (e.g. a vertex $\left(d^{\prime}=0\right.$ ), an entire edge $\left(d^{\prime}=1\right)$, an entire side $\left(d^{\prime}=2\right)$ etc.). If the mesh $\mathcal{T}$ is regular, the maps $F_{P}$ are assumed compatible between patches in the sense that

$$
\text { if } \bar{P} \cap \overline{P^{\prime}} \neq \emptyset:\left.F_{P}\right|_{\bar{P} \cap \overline{P^{\prime}}}=\left.F_{P^{\prime}}\right|_{\bar{P} \cap \overline{P^{\prime}}} \text {, i.e. } \quad F_{P}(x)=F_{P^{\prime}}(x) \quad \forall x \in \bar{P} \cap \overline{P^{\prime}} .
$$

The $\mathcal{T}_{P}$ are 1-irregular, if they consist of quadratics resp. hexagonal elements with at most one irregular ("hanging") node per side. $\mathcal{T}$ is 1-irregular, if the $\mathcal{T}_{P} \subset \mathcal{T}$ are either regular or 1-irregular and compatible between patches.

\subsubsection{Polynomial spaces}

On the reference element we define spaces of polynomials of degree $p \geq 0$ as follows:

$$
\begin{aligned}
& Q_{p}=\operatorname{span}\left\{\hat{x}^{\alpha}: 0 \leq \alpha_{i} \leq p, 1 \leq i \leq d\right\}, \\
& P_{p}=\operatorname{span}\left\{\hat{x}^{\alpha}: 0 \leq|\alpha| \leq p\right\} .
\end{aligned}
$$

\subsubsection{Polynomial subspaces on $\hat{P}$}

Let $\mathcal{T}$ be any mesh as in 2.1.1 and let

$$
\boldsymbol{p}=\left\{p_{K}: K \in \mathcal{T}\right\}
$$

be a polynomial degree vector on $\mathcal{T}$. The definition of a discontinuous $\boldsymbol{h} \boldsymbol{p}$-FE space is now straightforward: if $F_{\mathcal{P}}=\left\{F_{P}: P \in \mathcal{P}\right)$ denotes the patch-map vector, we set

$$
\begin{aligned}
S^{\mathbf{p}, 0}\left(\Omega, \mathcal{T}, F_{\mathcal{P}}\right):=\left\{u \in L_{2}(\Omega) \mid\right. & \left.u\right|_{K} \circ F_{K} \in Q_{p_{K}} \text { if } K \in \mathcal{T} \text { is quadrilateral } \\
& \text { resp. } \left.\left.u\right|_{K} \circ F_{K} \in P_{p_{K}} \text { if } K \text { is triangular }\right\} .
\end{aligned}
$$

No inter-element continuity is imposed here. If the polynomial degree is uniform, $p_{K}=p$ for all $K \in \mathcal{T}$, we write $S^{p, 0}\left(\Omega, \mathcal{T}, F_{\mathcal{P}}\right)$. If the choice of $\Omega, \mathcal{T}$ and $F_{\mathcal{P}}$ is clear from the context, we omit them and write $S^{p, 0}$. 
Let us now turn to continuous $\boldsymbol{h} \boldsymbol{p}$-FE spaces. Here we assume $\mathcal{T}$ to be either regular or 1-irregular. If the polynomial degrees $p_{K}$ are uniform, namely $p_{K}=p$ for all $K$, we define, for $p \geq 1$,

$$
S^{p, 1}\left(\Omega, \mathcal{T}, F_{\mathcal{P}}\right)=S^{p, 0}\left(\Omega, \mathcal{T}, F_{\mathcal{P}}\right) \cap H^{1}(\Omega)
$$

i.e. inter-element continuity is now enforced and the compatibility condition (2.5) between patches is required. If the polynomial degrees are nonuniform, there are several ways to enforce inter-element continuity - assume that $K, K^{\prime} \in \mathcal{T}$ share a $d-1$ dimensional set, and that $p_{K}<p_{K^{\prime}}$. One can now either enrich the polynomials on $K$ or constrain the polynomials on $K^{\prime}$. We adopt here the latter approach and set

$$
S^{\mathbf{p}, 1}\left(\Omega, \mathcal{T}, F_{\mathcal{P}}\right)=S^{\mathbf{p}, 0}\left(\Omega, \mathcal{T}, F_{\mathcal{P}}\right) \cap H^{1}(\Omega)
$$

Note that one could even allow anisotropic/nonuniform polynomial degrees within an element $K \in \mathcal{T}$ - this becomes important when adaptivity is considered (see [5] and the references therein). Definition (2.9) implies that the degrees of freedom from $K^{\prime}$ that are unmatched by those from $K$ are constrained to zero on interfaces $\bar{K} \cap \overline{K^{\prime}}$.

\subsection{The $h p$-SDFEM}

The $h p$-SDFEM approximation of (2.1) is defined as follows: find $u_{\mathrm{SD}} \in S^{\mathbf{p}, 1}$ such that

$$
\left(\mathcal{L} u_{\mathrm{SD}}, v+\delta \mathcal{L} v\right)+\left(u_{\mathrm{SD}}, v\right)_{\Gamma_{-}}=(f, v+\delta \mathcal{L} v)+(g, v)_{\Gamma_{-}} \quad \forall v \in S^{\mathbf{p}, 1}
$$

where $\delta$ is a positive piecewise constant function defined on the partition $\mathcal{T}$ (namely, $\delta$ is constant on each $K \in \mathcal{T})$. In $(2.10),(\cdot, \cdot)$ denotes the inner product of $L_{2}(\Omega)$, and

$$
(w, v)_{\Gamma_{-}}=\int_{\Gamma_{-}}|a \cdot n| w v d s
$$

with analogous definition of $(\cdot, \cdot)_{\Gamma_{+}}$and associated norms $\|\cdot\|_{\Gamma_{-}}$and $\|\cdot\|_{\Gamma_{+}}$. lemma.

Our first result concerns the stability of the $h p$-SDFEM and is expressed in the next

Lemma 2.2 Suppose that there exists a positive constant $c_{0}$ such that

$$
b(x)-\frac{1}{2} \nabla \cdot a(x) \geq c_{0}, \quad x \in \bar{\Omega} .
$$

Then $u_{\mathrm{SD}}$ obeys the bound

$$
\left\|\sqrt{\delta} \mathcal{L} u_{\mathrm{SD}}\right\|^{2}+c_{0}\left\|u_{\mathrm{SD}}\right\|^{2}+\left\|u_{\mathrm{SD}}\right\|_{\Gamma_{+}}^{2}+\frac{1}{2}\left\|u_{\mathrm{SD}}\right\|_{\Gamma_{-}}^{2} \leq\|\sqrt{\delta} f\|^{2}+\frac{1}{c_{0}}\|f\|^{2}+2\|g\|_{\Gamma_{-}}^{2} .
$$


Proof: Select $v=u_{\mathrm{SD}}$ in $(2.10)$ and note that

$$
\begin{aligned}
& \left(\mathcal{L} u_{\mathrm{SD}}, u_{\mathrm{SD}}\right)+\left(u_{\mathrm{SD}}, u_{\mathrm{SD}}\right)_{\Gamma_{-}} \\
& =\left(\left(b-\frac{1}{2} \nabla \cdot a\right) u_{\mathrm{SD}}, u_{\mathrm{SD}}\right)+\frac{1}{2}\left\|u_{\mathrm{SD}}\right\|_{\Gamma_{+}}^{2}+\frac{1}{2}\left\|u_{\mathrm{SD}}\right\|_{\Gamma_{-}}^{2} .
\end{aligned}
$$

Applying (2.11) here and using the Cauchy-Schwarz inequality on the right-hand side in (2.10) with $v=u_{\mathrm{SD}}$, the result follows.

Now we embark on the error analysis of (2.10). We begin by decomposing

$$
\begin{aligned}
u-u_{\mathrm{SD}} & =(u-\Pi u)+\left(\Pi u-u_{\mathrm{SD}}\right) \\
& \equiv \eta+\xi
\end{aligned}
$$

where $\Pi u$ is a suitable projection of $u$ into $S^{p, 1}$; for the time being the choice of the projector $\Pi$ is of no significance and will be deferred until later. First we shall derive a bound on $\xi$ in terms of $\eta$; the final error bound on $u-u_{\mathrm{SD}}$ will then follow from bounds on the projection error $\eta$.

Lemma 2.3 Assuming that (2.11) holds, and $u \in H^{1}(\Omega)$, we have that

$$
\|\sqrt{\delta} \mathcal{L} \xi\|^{2}+\|c \xi\|^{2}+\frac{1}{2}\|\xi\|_{\Gamma_{+}}^{2}+\|\xi\|_{\Gamma_{-}}^{2} \leq\left\|\sqrt{\delta} \mathcal{L} \eta-\frac{1}{\sqrt{\delta}} \eta\right\|^{2}+4\|c \eta\|^{2}+2\|\eta\|_{\Gamma_{+}}^{2},
$$

where $c \in C(\bar{\Omega})$ is defined by

$$
c^{2}(x)=b(x)-\frac{1}{2} \nabla \cdot a(x), x \in \bar{\Omega} .
$$

Proof: Define the bilinear form

$$
B(w, v)=(\mathcal{L} w, v+\delta \mathcal{L} v)+(w, v)_{\Gamma_{-}}
$$

for $w, v \in H^{1}(\Omega)$ and the linear form

$$
\ell(v)=(f, v+\delta \mathcal{L} v)+(g, v)_{\Gamma_{-}},
$$

for $v \in H^{1}(\Omega)$. Then, from (2.14),

$$
\begin{aligned}
B(\xi, \xi) & =B\left(u-u_{\mathrm{SD}}-\eta, \xi\right) \\
& =B(u, \xi)-B\left(u_{\mathrm{SD}}, \xi\right)-B(\eta, \xi) .
\end{aligned}
$$

Since $u$ and $u_{\mathrm{SD}}$ solve (2.1) and (2.10) respectively, it follows that

$$
B(u, \xi)-B\left(u_{\mathrm{SD}}, \xi\right)=B(u, \xi)-\ell(\xi)=0 .
$$

From (2.19) and (2.20) we have, 


$$
B(\xi, \xi)=-B(\eta, \xi)
$$

Applying (2.13) from the proof of Lemma 2.2 with $u_{\mathrm{SD}}$ replaced by $\xi$,

$$
\|\sqrt{\delta} \mathcal{L} \xi\|^{2}+\|c \xi\|^{2}+\frac{1}{2}\|\xi\|_{\Gamma_{+}}^{2}+\frac{1}{2}\|\xi\|_{\Gamma_{-}}^{2}=-B(\eta, \xi) .
$$

The rest of the proof is devoted to bounding $B(\eta, \xi)$. By partial integration,

$$
-B(\eta, \xi)=\left(\frac{1}{\sqrt{\delta}} \eta-\sqrt{\delta} \mathcal{L} \eta, \sqrt{\delta} \mathcal{L} \xi\right)-2\left(c^{2} \eta, \xi\right)-(\eta, \xi)_{\Gamma_{+}} \cdot
$$

Hence

$$
\begin{aligned}
-B(\eta, \xi) \leq \frac{1}{2} & \left\|\frac{1}{\sqrt{\delta}} \eta-\sqrt{\delta} \mathcal{L} \eta\right\|^{2}+\frac{1}{2}\|\sqrt{\delta} \mathcal{L} \xi\|^{2} \\
& +\frac{1}{2}\|c \xi\|^{2}+2\|c \eta\|^{2}+\frac{1}{4}\|\xi\|_{\Gamma_{+}}^{2}+\|\eta\|_{\Gamma_{+}}^{2} .
\end{aligned}
$$

Substituting (2.23) into (2.22) and multiplying the resulting inequality by 2 gives (2.15).

\subsection{The $h p$-DGFEM}

Given that $K$ is an element in the partition $\mathcal{T}$, we denote by $\partial K$ the union of open faces of $K$. This is non-standard notation in that $\partial K$ is a subset of the boundary of $K$. Let $x \in \partial K$ and suppose that $n(x)$ denotes the unit outward normal vector to $\partial K$ at $x$. With these conventions, we define the inflow and outflow parts of $\partial K$, respectively, by

$$
\begin{aligned}
& \partial_{-} K=\{x \in \partial K: a(x) \cdot n(x)<0\} \\
& \partial_{+} K=\{x \in \partial K: a(x) \cdot n(x) \geq 0\}
\end{aligned}
$$

For each $K \in \mathcal{T}$ and any $v \in H^{1}(K)$ we denote by $v^{+}$the interior trace of $v$ on $\partial K$ (the trace taken from within $K)$. Now consider an element $K$ such that the set $\partial_{-} K \backslash \Gamma_{-}$is nonempty; then for each $x \in \partial_{-} K \backslash \Gamma_{-}$(with the exception of a set of $(d-1)$ dimensional measure zero) there exists a unique element $K^{\prime}$, depending on the choice of $x$, such that $x \in \partial_{+} K^{\prime}$. This is illustrated in Figure 2.

Now suppose that $v \in H^{1}(K)$ for each $K \in \mathcal{T}$. If $\partial_{-} K \backslash \Gamma_{-}$is nonempty for some $K \in \mathcal{T}$, then we can also define the outer trace $v^{-}$of $v$ on $\partial_{-} K \backslash \Gamma_{-}$relative to $K$ as the inner trace $v^{+}$relative to those elements $K^{\prime}$ for which $\partial_{+} K^{\prime}$ has intersection with $\partial_{-} K \backslash \Gamma_{-}$of positive $(d-1)$-dimensional measure. We also introduce the jump of $v$ across $\partial_{-} K \backslash \Gamma_{-}$:

$$
[v]=v^{+}-v^{-}
$$

Let $\delta \in H^{1}(K)$ for each $K \in \mathcal{T}$, and suppose that $\delta$ is positive on each $K \in \mathcal{T}$. Typically, $\delta$ is chosen to be constant on each $K \in \mathcal{T}$, although we shall not require this for now. 


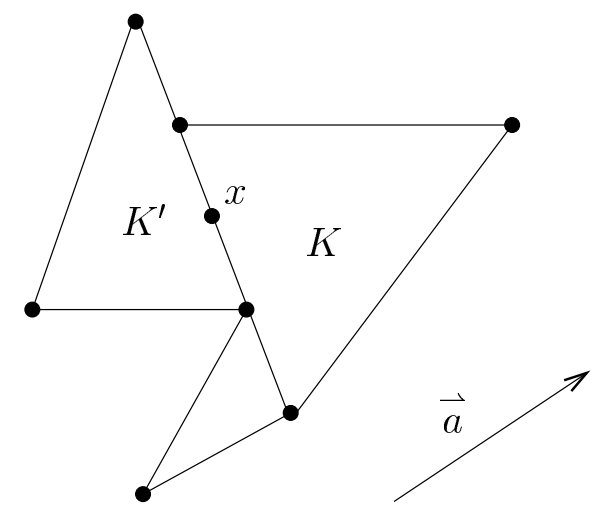

Figure 2: A point $x$ such that $x \in \partial_{-} K$ and $x \in \partial_{+} K^{\prime}$.

Suppose that $v, w \in H^{1}(K)$ for each $K \in \mathcal{T}$. We define

$$
\begin{aligned}
& B_{\mathrm{DG}}(w, v)=\sum_{K} \int_{K} \mathcal{L} w \cdot(v+\delta \mathcal{L} v) d x \\
& \quad-\sum_{K} \int_{\partial_{-} K \backslash \Gamma_{-}}(a \cdot n)[w] v^{+} d s-\sum_{K} \int_{\partial_{-} K \cap \Gamma_{-}}(a \cdot n) w^{+} v^{+} d s
\end{aligned}
$$

and put

$$
\ell_{\mathrm{DG}}(v)=\sum_{K} \int_{K} f \cdot(v+\delta \mathcal{L} v) d x-\sum_{K} \int_{\partial_{-} K_{\cap} \Gamma_{-}}(a \cdot n) g v^{+} d s
$$

The $h p$-DGFEM approximation of (2.1) is defined as follows: find $u_{\mathrm{DG}} \in S^{\mathbf{p}, 0}$ such that

$$
B_{\mathrm{DG}}\left(u_{\mathrm{DG}}, v\right)=\ell_{\mathrm{DG}}(v) \quad \forall v \in S^{\mathbf{p}, 0}
$$

Next we study the stability of the discrete problem (2.26).

Lemma 2.4 Suppose that there exists a positive constant $c_{0}$ such that (2.11) holds. Then $u_{\mathrm{DG}}$ obeys the bound

$$
\begin{aligned}
\sum_{K}\left\|\sqrt{\delta} \mathcal{L} u_{\mathrm{DG}}\right\|_{K}^{2}+c_{0}\left\|u_{\mathrm{DG}}\right\|_{K}^{2}+\sum_{K}\left\|u_{\mathrm{DG}}^{+}-u_{\mathrm{DG}}^{-}\right\|_{\partial_{-} K \backslash \Gamma_{-}}^{2} \\
\quad+\sum_{K}\left\|u_{\mathrm{DG}}^{+}\right\|_{\partial_{+} K \cap \Gamma_{+}}^{2}+\frac{1}{2} \sum_{K}\left\|u_{\mathrm{DG}}^{+}\right\|_{\partial_{-} K \cap \Gamma_{-}}^{2} \\
\leq \sum_{K}\|\sqrt{\delta} f\|_{K}^{2}+\frac{1}{c_{0}} \sum_{K}\|f\|_{K}^{2}+2 \sum_{K}\|g\|_{\partial_{-} K \cap \Gamma_{-}}^{2} .
\end{aligned}
$$


Remark: This bound is analogous to the estimate (2.12) for the $h p$-SDFEM.

Proof: Take $v=u_{\mathrm{DG}}$ in $(2.26)$; this gives

$$
B_{\mathrm{DG}}\left(u_{\mathrm{DG}}, u_{\mathrm{DG}}\right)=\ell_{\mathrm{DG}}\left(u_{\mathrm{DG}}\right) .
$$

We begin by bounding the left hand side in (2.28) from below. Upon partial integration, (2.24) gives

$$
\begin{aligned}
B_{\mathrm{DG}}\left(u_{\mathrm{DG}}, u_{\mathrm{DG}}\right)= & \sum_{K} \int_{K} \delta\left|\mathcal{L} u_{\mathrm{DG}}\right|^{2} d x \\
& +\sum_{K} \int_{K}\left(b-\frac{1}{2} \nabla \cdot a\right)\left|u_{\mathrm{DG}}\right|^{2} d x \\
& +\frac{1}{2} \sum_{K} \int_{\partial K}(a \cdot n)\left|u_{\mathrm{DG}}^{+}\right|^{2} d s \\
& -\sum_{K} \int_{\partial_{-} K \backslash \Gamma_{-}}(a \cdot n)\left[u_{\mathrm{DG}}\right] u_{\mathrm{DG}}^{+} d s \\
& -\sum_{K} \int_{\partial_{-}}(a \cdot n)\left|u_{\mathrm{DG}}^{+}\right|^{2} d s .
\end{aligned}
$$

Now decomposing $\partial K$ into the union of four disjoint sets

$$
\partial K=\left(\partial_{-} K \backslash \Gamma_{-}\right) \cup\left(\partial_{-} K \cap \Gamma_{-}\right) \cup\left(\partial_{+} K \backslash \Gamma_{+}\right) \cup\left(\partial_{+} K \cap \Gamma_{+}\right)
$$

and writing

$$
\left[u_{\mathrm{DG}}\right] u_{\mathrm{DG}}^{+}=\left(u_{\mathrm{DG}}^{+}-u_{\mathrm{DG}}^{-}\right) u_{\mathrm{DG}}^{+}=\frac{1}{2}\left|u_{\mathrm{DG}}^{+}\right|^{2}+\frac{1}{2}\left(u_{\mathrm{DG}}^{+}-u_{\mathrm{DG}}^{-}\right)^{2}-\frac{1}{2}\left|u_{\mathrm{DG}}^{-}\right|^{2},
$$

the last three terms in (2.29) can be rewritten as

$$
\begin{aligned}
& \frac{1}{2} \sum_{K} \int_{\partial_{-} K \cap \Gamma_{-}}-(a \cdot n)\left|u_{\mathrm{DG}}^{+}\right|^{2} d s \\
& \quad+\frac{1}{2} \sum_{K} \int_{\partial_{-} \backslash \Gamma_{-}}-(a \cdot n)\left|u_{\mathrm{DG}}^{+}-u_{\mathrm{DG}}^{-}\right|^{2} d s \\
& \quad+\frac{1}{2} \sum_{K} \int_{\partial_{+}}(a \cdot n)\left|u_{\mathrm{DG}}^{+}\right|^{2} d s .
\end{aligned}
$$

Here we made use of the fact that

$$
\sum_{K} \int_{\partial_{+} K \backslash \Gamma_{+}}(a \cdot n)\left|u_{\mathrm{DG}}^{+}\right|^{2} d s+\sum_{K} \int_{\partial_{-} K \backslash \Gamma_{-}}(a \cdot n)\left|u_{\mathrm{DG}}^{-}\right|^{2} d s=0 .
$$


Using (2.30) in (2.29) yields

$$
\begin{aligned}
B_{\mathrm{DG}}\left(u_{\mathrm{DG}}, u_{\mathrm{DG}}\right) \geq & \sum_{K}\left\|\sqrt{\delta} \mathcal{L} u_{\mathrm{DG}}\right\|_{K}^{2} \\
& +c_{0} \sum_{K}\left\|u_{\mathrm{DG}}\right\|_{K}^{2}+\frac{1}{2} \sum_{K}\left\|u_{\mathrm{DG}}^{+}\right\|_{\partial_{-} K \cap \Gamma_{-}}^{2} \\
& +\frac{1}{2} \sum_{K}\left\|u_{\mathrm{DG}}^{+}-u_{\mathrm{DG}}^{-}\right\|_{\partial_{-} K \backslash \Gamma_{-}}^{2} \\
& +\frac{1}{2} \sum_{K}\left\|u_{\mathrm{DG}}^{+}\right\|_{\partial_{+} K \cap \Gamma_{+}}^{2} .
\end{aligned}
$$

Now we bound the right-hand side in (2.28) using (2.25):

$$
\begin{aligned}
\left|\ell_{\mathrm{DG}}\left(u_{\mathrm{DG}}\right)\right| \leq & \sum_{K}\|f\|_{K}\left\|u_{\mathrm{DG}}\right\|_{K} \\
& +\sum_{K}\|\sqrt{\delta} f\|_{K}\left\|\sqrt{\delta} \mathcal{L} u_{\mathrm{DG}}\right\|_{K} \\
& +\sum_{K}\|g\|_{\partial_{-} K \cap \Gamma_{-}}\left\|u_{\mathrm{DG}}^{+}\right\|_{\partial_{-} K \cap \Gamma_{-}} \\
\leq & \frac{c_{0}}{2} \sum_{K}\left\|u_{\mathrm{DG}}\right\|_{K}^{2}+\frac{1}{2 c_{0}} \sum_{K}\|f\|_{K}^{2} \\
& +\frac{1}{2} \sum_{K}\left\|\sqrt{\delta} \mathcal{L} u_{\mathrm{DG}}\right\|_{K}^{2}+\frac{1}{2} \sum_{K}\|\sqrt{\delta} f\|_{K}^{2} \\
& +\frac{1}{4} \sum_{K}\left\|u_{\mathrm{DG}}^{+}\right\|_{\partial_{-} K \cap \Gamma_{-}}^{2}+\sum_{K}\|g\|_{\partial_{-} K \cap \Gamma_{-}}^{2} .
\end{aligned}
$$

Inserting (2.31) and (2.32) into (2.28) gives (2.27).

We now discuss the error analysis of $h p$-DGFEM. We write

$$
\begin{aligned}
u-u_{\mathrm{DG}} & =(u-\Pi u)+\left(\Pi u-u_{\mathrm{DG}}\right) \\
& \equiv \eta+\xi
\end{aligned}
$$

where $\Pi u$ is a suitable projection of $u$ into $S^{\mathbf{p}, 0}$, to be chosen below.

Lemma 2.5 Assuming that (2.11) holds and $u \in H^{1}(K)$ for each $K \in \mathcal{T}$. We have that

$$
\begin{aligned}
& \sum_{K}\|\sqrt{\delta} \mathcal{L} \xi\|_{K}^{2}+\sum_{K}\|c \xi\|_{K}^{2}+\sum_{K}\left\|\xi^{+}\right\|_{\partial_{-} K \cap \Gamma_{-}}^{2} \\
& \quad+\frac{1}{2} \sum_{K}\left\|\xi^{+}\right\|_{\partial_{+} K \cap \Gamma_{+}}^{2}+\frac{1}{2} \sum_{K}\left\|\xi^{+}-\xi^{-}\right\|_{\partial_{-} K \backslash \Gamma_{-}}^{2} \\
& \leq \sum_{K}\left\|\sqrt{\delta} \mathcal{L} \eta-\frac{1}{\sqrt{\delta}} \eta\right\|_{K}^{2}+4 \sum_{K}\|c \eta\|_{K}^{2} \\
& \quad+2 \sum_{K}\left\|\eta^{+}\right\|_{\partial_{+} K \cap \Gamma_{+}}^{2}+\sum_{K}\left\|\eta^{-}\right\|_{\partial_{-} K \backslash \Gamma_{-}}^{2} .
\end{aligned}
$$


Proof As in Lemma 2.3,

$$
B_{\mathrm{DG}}(\xi, \xi)=-B_{\mathrm{DG}}(\eta, \xi)
$$

Applying (2.31) with $u_{\mathrm{DG}}$ replaced by $\xi$ gives

$$
\begin{aligned}
& \sum_{K}\|\sqrt{\delta} \mathcal{L} \xi\|_{K}^{2}+\sum_{K}\|c \xi\|_{K}^{2}+\frac{1}{2} \sum_{K}\left\|\xi^{+}\right\|_{\partial_{-} K \cap \Gamma_{-}}^{2} \\
& \quad+\frac{1}{2} \sum_{K}\left\|\xi^{+}\right\|_{\partial_{+} K \cap \Gamma_{+}}^{2}+\frac{1}{2} \sum_{K}\left\|\xi^{+}-\xi^{-}\right\|_{\partial_{-} K \backslash \Gamma_{-}}^{2} \leq\left|B_{\mathrm{DG}}(\eta, \xi)\right| .
\end{aligned}
$$

Next we transform $B_{\mathrm{DG}}(\eta, \xi)$ :

$$
\begin{aligned}
B_{\mathrm{DG}}(\eta, \xi)= & \sum_{K} \int_{K} \delta \mathcal{L} \eta \cdot \mathcal{L} \xi d x \\
& +2 \sum_{K} \int_{K}\left(b-\frac{1}{2} \nabla \cdot a\right) \eta \xi d x \\
& -\sum_{K} \int_{K} \eta \mathcal{L} \xi d x+\sum_{K} \int_{\partial_{+} K \cap \Gamma_{+}}(a \cdot n) \eta^{+} \xi^{+} d s \\
& +\sum_{K} \int_{\partial_{+}}(a \cdot n) \eta^{+} \xi^{+} d s+\sum_{K} \int_{\partial_{-}}\left(a \cdot n \backslash \Gamma_{+}\right.
\end{aligned}
$$

Now

$$
\begin{aligned}
& \left|\sum_{K} \int_{\partial_{+} K \backslash \Gamma_{+}}(a \cdot n) \eta^{+} \xi^{+} d s+\sum_{K} \int_{\partial_{-} K \backslash \Gamma_{-}}(a \cdot n) \eta^{-} \xi^{+} d s\right| \\
& \quad \leq \sum_{K}\left\|\eta^{-}\right\|_{\partial_{-} K \backslash \Gamma_{-}}\left\|\xi^{+}-\xi^{-}\right\|_{\partial_{-} K \backslash \Gamma_{-}} \\
& \quad \leq \frac{1}{4} \sum_{K}\left\|\xi^{+}-\xi^{-}\right\|_{\partial_{-} K \backslash \Gamma_{-}}^{2}+\sum_{K}\left\|\eta^{-}\right\|_{\partial_{-} K \backslash \Gamma_{-}}^{2} .
\end{aligned}
$$

Substituting (2.37) into (2.36), we get

$$
\begin{aligned}
B_{\mathrm{DG}}(\eta, \xi) \leq & \frac{1}{2} \sum_{K}\|\sqrt{\delta} \mathcal{L} \xi\|_{K}^{2}+\frac{1}{2} \sum_{K}\left\|\sqrt{\delta} \mathcal{L} \eta-\frac{1}{\sqrt{\delta}} \eta\right\|_{K}^{2} \\
& +\frac{1}{2} \sum_{K}\|c \xi\|_{K}^{2}+2 \sum_{K}\|c \eta\|_{K}^{2} \\
& +\frac{1}{4} \sum_{K}\left\|\xi^{+}\right\|_{\partial_{+} K \cap \Gamma_{+}}^{2}+\sum_{K}\left\|\eta^{+}\right\|_{\partial_{+} K \cap \Gamma_{+}}^{2} \\
& +\frac{1}{4} \sum_{K}\left\|\xi^{+}-\xi^{-}\right\|_{\partial_{-} K \backslash \Gamma_{-}}^{2}+\sum_{K}\left\|\eta^{-}\right\|_{\partial_{-} K \backslash \Gamma_{-}}^{2} .
\end{aligned}
$$

Now inserting (2.38) into (2.35) gives (2.34). 


\section{$3 \quad h p$-Error Estimates}

In this section, we shall construct the $h p$-approximation projector $\Pi$ in the error estimates (2.14), (2.33) and derive $h p$-error bounds for the $h p$-SDFEM as well as for the $h p$-DGFEM introduced in the previous section. The bounds are explicit in $h$ and $p$ and in the regularities of the solution and allow us to deduce in particular exponential convergence estimates for piecewise analytic solutions. For simplicity, we restrict ourselves to $d=2$ space dimensions and to meshes consisting of quadrilateral elements.

\subsection{One-dimensional $h p$-approximation}

We cite some approximation results from [10]. To this end, we set $\hat{I}=(-1,1)$ and denote by $\|u\|_{k, \hat{I}}$ resp. $|u|_{k, \hat{I}}$ the $H^{k}(\hat{I})$ norm resp. seminorm on $\hat{I}$. Denote further $S^{p}(\hat{I})$ the polynomials of degree $p$ on $\hat{I}$. Then we have

Theorem 3.6 Let $u \in H^{k+1}(\hat{I})$ for some $k \geq 0$. Then, for every $p \geq 1$, there exists $\pi_{p} u \in S^{p}(\hat{I})$ such that

$$
\left\|u^{\prime}-\left(\pi_{p} u\right)^{\prime}\right\|_{\hat{I}}^{2} \leq \frac{(p-s) !}{(p+s) !}|u|_{s+1, \hat{I}}^{2}
$$

for any $0 \leq s \leq \min (p, k)$ and such that

$$
\left\|u-\pi_{p} u\right\|_{\hat{I}}^{2} \leq \frac{1}{p(p+1)} \frac{(p-t) !}{(p+t) !}|u|_{t+1, \hat{I}}^{2}
$$

for any $0 \leq t \leq \min (p, k)$. Moreover, we have

$$
\pi_{p} u( \pm 1)=u( \pm 1)
$$

For the proof, we refer e.g. to [10].

Corollary 3.6A The projector $\pi_{p}$ whose existence is asserted in Theorem 3.6 is bounded as follows:

$$
\begin{gathered}
\left\|\left(\pi_{p} u\right)^{\prime}\right\|_{\hat{I}} \leq 2\left\|u^{\prime}\right\|_{\hat{I}}, \\
\left\|\pi_{p} u\right\|_{\hat{I}} \leq\|u\|_{\hat{I}}+\frac{1}{\sqrt{p(p+1)}}\left\|u^{\prime}\right\|_{\hat{I}}
\end{gathered}
$$

for all $p \geq 1$ and every $u \in H^{1}(\hat{I})$.

Proof: The inequality (3.1) with $s=0$ implies

$$
\left\|\left(\pi_{p} u\right)^{\prime}\right\|_{\hat{I}} \leq\left\|\left(\pi_{p} u\right)^{\prime}-u^{\prime}\right\|_{\hat{I}}+\left\|u^{\prime}\right\|_{\hat{I}} \leq 2\left\|u^{\prime}\right\|_{\hat{I}} .
$$

Similarly, (3.2) with $t=0$ implies

$$
\begin{aligned}
\left\|\pi_{p} u\right\|_{\hat{I}} & \leq\left\|\pi_{p} u-u\right\|_{\hat{I}}+\|u\|_{\hat{I}} \\
& \leq\|u\|_{\hat{I}}+\frac{1}{\sqrt{p(p+1)}}\left\|u^{\prime}\right\|_{\hat{I}} .
\end{aligned}
$$




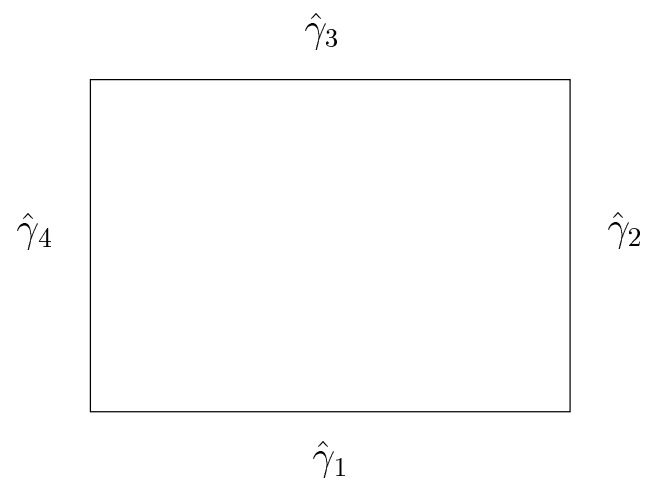

Figure 3: $\hat{Q}$ and the notation for the sides.

\subsection{Approximation on quadrilaterals}

Higher dimensional approximation results will be obtained from Theorem 3.6 by tensor product construction. We denote by $\pi_{p}^{i} u$ the one-dimensional projector in Theorem 3.6 applied to $u$ as function of the $i$ th coordinate alone and perform the error analysis for $d=2$.

Let $\hat{Q}=(-1,1)^{2}$ and denote by $\hat{\gamma}_{i}, i=1,2,3,4$, the sides of $\hat{Q}$ as shown in Figure 3 .

Theorem 3.7 (Reference Element Approximation)

Let $\hat{Q}=(-1,1)^{2}$, as in Figure 3, $p \geq 1$ and assume that $u \in H^{k+1}(\hat{Q})$ for some $k \geq 1$. Let $\Pi_{p}=\pi_{p}^{1} \pi_{p}^{2}$ denote the tensor product projector. Then there holds:

$$
\begin{gathered}
\Pi_{p} u=u \text { at the vertices of } \hat{Q}, \\
\left.\Pi_{p} u\right|_{\hat{\gamma}_{i}}= \begin{cases}\pi_{p}^{1}\left(\left.u\right|_{\hat{\gamma}_{i}}\right) & \text { if } i \text { is odd }, \\
\pi_{p}^{2}\left(\left.u\right|_{\hat{\gamma}_{i}}\right) & \text { if } i \text { is even } .\end{cases}
\end{gathered}
$$

The following error estimates hold:

$$
\begin{aligned}
\left\|\nabla\left(u-\Pi_{p} u\right)\right\|_{\hat{Q}}^{2} \leq & 2 \frac{(p-s) !}{(p+s) !}\left\{\left\|\partial_{1}^{s+1} u\right\|_{\hat{Q}}^{2}+\left\|\partial_{2}^{s+1} u\right\|_{\hat{Q}}^{2}\right\} \\
& +\frac{8}{p(p+1)} \frac{(p-s+1) !}{(p+s-1) !}\left\{\left\|\partial_{1}^{s} \partial_{2} u\right\|_{\hat{Q}}^{2}+\left\|\partial_{1} \partial_{2}^{s} u\right\|_{\hat{Q}}^{2}\right\} \\
\left\|u-\Pi_{p} u\right\|_{\hat{Q}}^{2} \leq & \frac{2}{p(p+1)} \frac{(p-s) !}{(p+s) !}\left\{\left\|\partial_{1}^{s+1} u\right\|_{\hat{Q}}^{2}+2\left\|\partial_{2}^{s+1} u\right\|_{\hat{Q}}^{2}\right\} \\
& +\frac{4}{p^{2}(p+1)^{2}} \frac{(p-s+1) !}{(p+s-1) !}\left\|\partial_{1} \partial_{2}^{s} u\right\|_{\hat{Q}}^{2},
\end{aligned}
$$


for any $0 \leq s \leq \min (p, k)$.

Proof: We prove (3.9). Clearly,

$$
\left\|u-\Pi_{p} u\right\|_{\hat{Q}}^{2} \leq 2\left\|u-\pi_{p}^{1} u\right\|_{\hat{Q}}^{2}+2\left\|\pi_{p}^{1}\left(u-\pi_{p}^{2} u\right)\right\|_{\hat{Q}}^{2} .
$$

For the first term we use the bound (3.2), resulting in

$$
\left\|u-\pi_{p}^{1} u\right\|_{\hat{Q}}^{2} \leq \frac{1}{p(p+1)} \frac{(p-s) !}{(p+s) !}\left\|\partial_{1}^{s+1} u\right\|_{\hat{Q}}^{2} .
$$

For the second term, (3.5) and (3.2) give

$$
\begin{aligned}
& \left\|\pi_{p}^{1}\left(u-\pi_{p}^{2} u\right)\right\|_{\hat{Q}}^{2} \leq 2\left\|u-\pi_{p}^{2} u\right\|_{\hat{Q}}^{2}+\frac{2}{p(p+1)}\left\|\partial_{1}\left(u-\pi_{p}^{2} u\right)\right\|_{\hat{Q}}^{2} \\
& \quad \leq \frac{2}{p(p+1)} \frac{(p-t) !}{(p+t) !}\left\|\partial_{2}^{t+1} u\right\|_{\hat{Q}}^{2}+\frac{2}{p^{2}(p+1)^{2}} \frac{(p-r) !}{(p+r) !}\left\|\partial_{1} \partial_{2}^{r+1} u\right\|_{\hat{Q}}^{2}
\end{aligned}
$$

Selecting $t=s$ and $r=s-1$ gives (3.9). The proof of (3.8) is analogous.

\subsection{Approximation on quadrilateral meshes with hanging nodes}

Consider now a mesh patch $P \in \mathcal{P}$ with mesh $\mathcal{T}_{P}$ and corresponding reference mesh $\hat{\mathcal{T}}_{P}$ in $\hat{P}$. We assume that all $K \in \mathcal{T}_{P}$ are quadrilateral, possibly with hanging nodes. With $K$ we associate the edge-lengths of the sides of $\hat{K}=F_{P}^{-1}(K)$ denoted by $h_{i, \hat{K}}, i=1,2$.

\section{Theorem 3.8 (Discontinuous Approximation)}

Let $P \in \mathcal{P}$ with quadrilateral, possibly 1-irregular mesh $\mathcal{T}_{P}$ of shape-regular elements and polynomial degree distribution $\mathbf{p}$. For all $K \in \mathcal{T}_{P}$ let $\left.u\right|_{K} \in H^{k_{K}+1}(K)$ for some $k_{K} \geq 1$ and define $\Pi u \in S^{\mathbf{p}, 0}\left(P, \mathcal{T}_{P}\right)$ element-wise by

$$
\left.(\Pi u)\right|_{K} \circ F_{P}:=\Pi_{p_{K}}\left(\left.u\right|_{K} \circ F_{P}\right) \quad \forall K \in \mathcal{T}_{P},
$$

with $\Pi_{p}$ as in Theorem 3.7.

Then, for $p_{K} \geq 1$ and for $0 \leq s_{K} \leq \min \left(p_{K}, k_{K}\right)$ the following estimate holds:

$$
\|u-\Pi u\|_{P}^{2} \leq C \sum_{K \in \mathcal{T}_{P}}\left(\frac{h_{K}}{2}\right)^{2 s_{K}+2} \frac{1}{p_{K}\left(p_{K}+1\right)} \Phi\left(p_{K}, s_{K}\right)|\hat{u}|_{s_{K}+1, \hat{K}}^{2},
$$

where $\hat{u}=u \circ F_{P}, K=F_{P}(\hat{K})$ and

$$
\Phi(p, s):=\frac{(p-s) !}{(p+s) !}+\frac{1}{p(p+1)} \frac{(p-s+1) !}{(p+s-1) !}, 0 \leq s \leq p .
$$


Furthermore,

$$
\|\nabla(u-\Pi u)\|_{P}^{2} \leq C \sum_{K \in \mathcal{T}_{P}}\left(\frac{h_{K}}{2}\right)^{2 s_{K}} \Phi\left(p_{K}, s_{K}\right)|\hat{u}|_{s_{K}+1, \hat{K}}^{2}
$$

The constant $C>0$ in these estimates depends only on $F_{P}$, but is independent of $h_{K}, p_{K}$ and $s_{K}$.

Proof: The $L^{2}$-estimate (3.10) follows immediately by a change of variables and a scaling argument from Theorem 3.7.

For the gradient estimate, we observe that

$$
\|\nabla(u-\Pi u)\|_{P} \leq C\left(F_{P}\right)\left\|\hat{\nabla}\left((u-\Pi u) \circ F_{P}\right)\right\|_{\hat{P}} .
$$

For the right-hand side we use (3.8), after scaling to the reference element:

$$
\begin{aligned}
& \left\|\hat{\nabla}\left((u-\Pi u) \circ F_{P}\right)\right\|_{\hat{P}}^{2} \\
& =\sum_{\hat{K} \in \hat{\mathcal{T}}_{P}}\left\|\hat{\partial}_{1}\left((u-\Pi u) \circ F_{P}\right)\right\|_{\hat{K}}^{2}+\left\|\hat{\partial}_{2}\left((u-\Pi u) \circ F_{P}\right)\right\|_{\hat{K}}^{2} \\
& =\frac{1}{4} \sum_{\substack{\hat{K} \in \hat{\mathcal{T}}_{P} \\
i=1,2}} h_{1, \hat{K}} h_{2, \hat{K}}\left\|\hat{\partial}_{i}\left(I-\Pi_{p_{K}}\right) u \circ F_{P} \circ A_{K}\right\|_{\hat{Q}}^{2} \\
& \stackrel{(3.8)}{\leq} \frac{1}{2}\left(h_{\hat{K}}\right)^{2} \sum_{\hat{K} \in \hat{\mathcal{T}}_{P}}\left\{\frac{\left(p_{K}-s_{K}\right) !}{\left(p_{K}+s_{K}\right) !}\left(\left\|\hat{\partial}_{1}^{s_{K}+1} u_{0, K}\right\|_{\hat{Q}}^{2}+\left\|\hat{\partial}_{2}^{s_{K}+1} u_{0, K}\right\|_{\hat{Q}}^{2}\right)\right. \\
& \left.+\frac{4}{p_{K}\left(p_{K}+1\right)} \frac{\left(p_{K}-s_{K}+1\right) !}{\left(p_{K}+s_{K}-1\right) !}\left(\left\|\hat{\partial}_{1}^{s_{K}} \hat{\partial}_{2} u_{0, K}\right\|_{\hat{Q}}^{2}+\left\|\hat{\partial}_{1} \hat{\partial}_{2}^{s_{K}} u_{0, K}\right\|_{\hat{Q}}^{2}\right)\right\},
\end{aligned}
$$

where

$$
u_{0, K}:=u \circ F_{P} \circ A_{K}=\hat{u} \circ A_{K}, \quad K \in \mathcal{T}_{P} .
$$

Affine scaling from $\hat{Q}$ to $\hat{K} \in \hat{\mathcal{T}}_{P}$ and noting (2.4) gives the assertion.

The error bounds in Theorem 3.8 simplify for uniform $p$.

Corollary 3.8A (Uniform order estimate) Assume that $\hat{u}:=u \circ F_{P} \in H^{k+1}(\hat{P})$ and that for all $K \in \mathcal{T}_{P}$

$$
p_{K}=p \geq 1, \quad s_{K}=s, \quad 0 \leq s \leq \min (p, k) .
$$

Then, for $\Pi u \in S^{p, 0}\left(P, \mathcal{T}_{P}\right)$ and $\hat{u}:=u \circ F_{P}$, the following estimates hold:

$$
\|u-\Pi u\|_{P}^{2} \leq C \frac{1}{p(p+1)} \Phi(p, s) \sum_{K \in \mathcal{T}_{P}}\left(\frac{h_{K}}{2}\right)^{2 s+2}|\hat{u}|_{s+1, \hat{K}}^{2},
$$


and

$$
\|\nabla(u-\Pi u)\|_{P}^{2} \leq C \Phi(p, s) \sum_{K \in \mathcal{T}_{P}}\left(\frac{h_{K}}{2}\right)^{2 s}|\hat{u}|_{s+1, \hat{K}}^{2}
$$

Here $C>0$ is a constant that depends only on the patch mapping $F_{P}$ but not on $s, p, h_{K}$.

Remark (Anisotropic error estimates)

We note in passing that the above error estimate assumed the shape regularity of the $\hat{K}$ merely for convenience - in fact the explicit error bounds in Theorem 3.7 and 3.8 above could be easily generalized to anisotropic element shapes (with edge-lengths $h_{1 K}$ and $h_{2 K}$ ) and even to anisotropic polynomial degrees $p_{1 K}, p_{2 K}$, say. Error bounds explicit in these parameters can be deduced by inspecting the proofs of the above theorems.

Theorem 3.8 addressed only discontinuous approximations; it turns out, however, that also continuous, piecewise polynomial approximations can be obtained.

\section{Theorem 3.9 (Continuous approximations)}

Let $\Omega \subset \mathbb{R}^{2}$ and let $P \in \mathcal{P}$ with a 1-irregular mesh consisting of shape regular quadrilaterals $K$ of diameter $h_{K}$. Let the polynomial degree be uniform, $p_{K}=p \geq 1$. Let $\left.u\right|_{K} \in H^{k_{K}+1}(K)$ for some $k_{K} \geq 1$ and let $u \in H^{2}(P)$.

Then there exists a projector $\widetilde{\Pi} u \in S^{p, 1}\left(P, \mathcal{T}_{P}\right)$ such that the error bounds (3.13), (3.14) hold, with a possibly different value of $C$.

Proof If $\mathcal{T}_{P}$ does not contain hanging nodes, $\mathcal{T}_{P}$ is regular and we take $\widetilde{\Pi}=\Pi$ in Theorem 3.8. Since $\Pi$ was constructed element-wise, the properties (3.10), (3.12) together with the assumption that $u \in H^{2}(P)$ give the continuity of $\Pi u$ in $\bar{P}$.

Suppose now that $\mathcal{T}_{P}$ contains hanging nodes. A typical situation in the reference mesh $\hat{\mathcal{T}}_{P}$ is shown in Figure 4 where the elements have been scaled to unit size for convenience.

Since $u \in H^{2}(P)$, also $u \in C^{0}(\bar{P})$. By (3.6), $u-\Pi u$ vanishes at the points $\times$ in Figure 4. Denote by $[u-\Pi u]_{i j}$ the jump of $u-\Pi u$ across $\gamma_{i j}$. By (3.6), the jump of $\Pi u$ across $\gamma_{23}$ is zero. Since $u \in C^{0}(\bar{P}),[u-\Pi u]_{i j}=-[\Pi u]_{i j}$. Further, $[\Pi u]_{i j} \in P_{p}\left(\gamma_{i j}\right)$.

We now construct a trace-lifting of $[\Pi u]$ across $\gamma_{12} \cup \gamma_{13}$ as follows: we set

$$
V(\xi)=-\left(\xi_{2}+1\right)\left\{\begin{array}{lll}
{[\Pi u]_{12}\left(\xi_{1}\right)} & \text { on } & \hat{K}_{2}, \\
{[\Pi u]_{13}\left(\xi_{1}\right)} & \text { on } & \hat{K}_{3} .
\end{array}\right.
$$

Since $[\Pi u]_{23}=0, V$ is continuous on $\overline{\hat{K}_{2} \cup \hat{K}_{3}}$ and

$$
\|\nabla V\|_{\hat{K}_{2} \cup \hat{K}_{3}} \leq C\|[\Pi u]\|_{H^{\frac{1}{2}}\left(\gamma_{12} \cup \gamma_{13}\right)},
$$




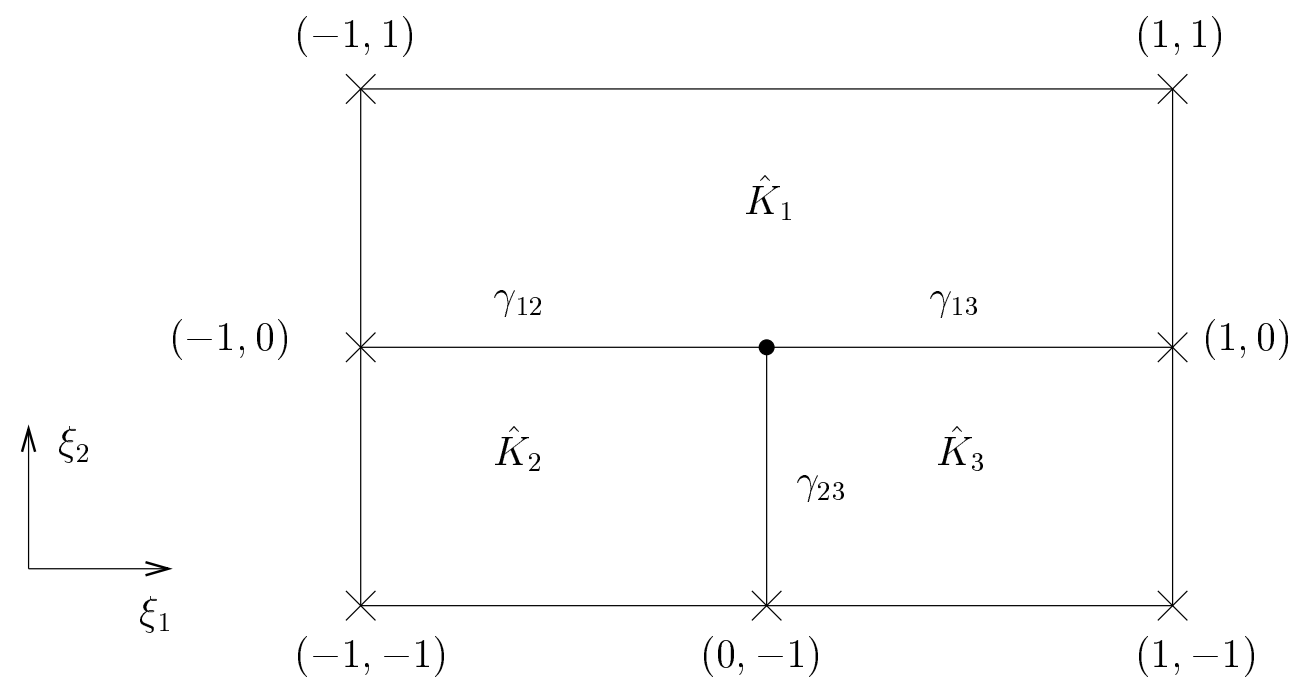

Figure 4: Hanging node $\bullet$ and adjacent elements.

where $C$ is independent of $p$. By the trace theorem and since $u \in C^{0}\left(\bigcup_{1}^{3} \overline{\hat{K}_{i}}\right)$, we have

$$
\begin{aligned}
\|[\Pi u]\|_{H^{\frac{1}{2}\left(\gamma_{12} \cup \gamma_{13}\right)}} & =\|[u-\Pi u]\|_{H^{\frac{1}{2}\left(\gamma_{12} \cup \gamma_{13}\right)}} \\
& \leq\left\|(u-\Pi u)^{+}\right\|_{H^{\frac{1}{2}}\left(\gamma_{12} \cup \gamma_{13}\right)}+\left\|(u-\Pi u)^{-}\right\|_{H^{\frac{1}{2}}\left(\gamma_{12} \cup \gamma_{13}\right)} \\
& \leq C \sum_{i=1}^{3}\|u-\Pi u\|_{H^{1}\left(\hat{K}_{i}\right)},
\end{aligned}
$$

where $(\cdot)^{ \pm}$denote traces from $\xi_{2}>0$ and $\xi_{2}<0$, respectively. We define

$$
\widetilde{\Pi} u:=\left\{\begin{array}{lll}
\Pi u & \text { on } & \hat{K}_{1} \\
V+\Pi u & \text { on } & \hat{K}_{2} \cup \hat{K}_{3} .
\end{array}\right.
$$

Now $\widetilde{\Pi} u$ is continuous on and across $\gamma_{12}$ and $\gamma_{13}$. Therefore, on $\hat{K}:=\overline{\hat{K}_{1} \cup \hat{K}_{2} \cup \hat{K}_{3}}$, we have that

$$
\|\nabla(u-\widetilde{\Pi} u)\|_{\hat{K}} \leq\|\nabla V\|_{\hat{K}_{2} \cup \hat{K}_{3}}+\sum_{i=1}^{3}\|\nabla(u-\Pi u)\|_{\hat{K}_{i}} .
$$

Using (3.15), (3.16) we get

$$
\|\nabla(u-\widetilde{\Pi} u)\|_{\hat{K}}^{2} \leq C \sum_{i=1}^{3}\|u-\Pi u\|_{1, \hat{K}_{i}}^{2},
$$

where $C>0$ is independent of $p$. 
Now suppose that the $\hat{K}_{i}, i=1,2,3$, are not of unit size but that their diameters are proportional to $h_{\hat{K}}$, where $h_{\hat{K}}$ in the diameter of $\hat{K}$. Performing a scaling of the independent variable by a factor of $h_{\hat{K}}$ in the estimate $(3.17)$ (noting that $\operatorname{diam}\left(\hat{K}_{2}\right)=\operatorname{diam}\left(\hat{K}_{3}\right)=h_{\hat{K}} / 2$, $\left.\operatorname{diam}\left(\hat{K}_{1}\right) \in\left[h_{\hat{K}} / 2, h_{\hat{K}}\right]\right)$, recalling (2.4) and inserting (3.13) and (3.14) into the resulting right-hand side, we deduce that a bound analogous to (3.14) holds with $\Pi$ replaced by $\widetilde{\Pi}$.

Concerning the analogue of the bound (3.13) with $\Pi$ replaced by $\widetilde{\Pi}$ and $\widetilde{\Pi}$ defined as above, again, we consider the configuration shown in Figure 4 with the elements scaled to unit size, for convenience. Observe that

$$
\begin{aligned}
\|V\|_{\hat{K}_{2} \cup \hat{K}_{3}} & \leq C\|[\Pi u-u]\|_{L_{2}\left(\gamma_{12} \cup \gamma_{13}\right)} \\
& \leq C\left(\left\|(u-\Pi u)^{+}\right\|_{L_{2}\left(\gamma_{12} \cup \gamma_{13}\right)}+\left\|(u-\Pi u)^{-}\right\|_{L_{2}\left(\gamma_{12} \cup \gamma_{13}\right)}\right) \\
& \leq C \sum_{i=1}^{3}\left(\|u-\Pi u\|_{\hat{K}_{i}}+\|u-\Pi u\|_{\hat{K}_{i}}^{1 / 2}\|\nabla(u-\Pi u)\|_{\hat{K}_{i}}^{1 / 2}\right)
\end{aligned}
$$

where in the transition to the last line we made use of the multiplicative trace inequality. Consequently, also

$$
\|u-\widetilde{\Pi} u\|_{\hat{K}} \leq C \sum_{i=1}^{3}\left(\|u-\Pi u\|_{\hat{K}_{i}}+\|u-\Pi u\|_{\hat{K}_{i}}^{1 / 2}\|\nabla(u-\Pi u)\|_{\hat{K}_{i}}^{1 / 2}\right) .
$$

Now suppose the $\hat{K}_{i}, i=1,2,3$, are of size proportional to $h_{\hat{K}}$; then, we may scale the independent variable by $h_{\hat{K}}$ in estimate (3.18) and insert (3.13) and (3.14) into the resulting right-hand side to deduce that a bound analogous to $(3.13)$ holds with $\Pi$ replaced by $\widetilde{\Pi}$.

Finally, we note that since

$$
\left.\widetilde{\Pi} u\right|_{\partial \hat{K}}=\Pi u_{\partial \hat{K}}
$$

further liftings in the presence of additional hanging nodes on $\partial \hat{K}$ can be performed in the adjacent element patches, resulting in the error bounds (3.13), (3.14) with a larger $C$.

\section{4 $h p$-Error Analysis of the DG- and the SDFEM}

We are now in a position to present error estimates for both the SD- and the DGFEM. We shall use the following norm defined by

$$
\begin{aligned}
\|u\| \|_{\mathrm{DG}}^{2} & :=\sum_{K \in \mathcal{T}}\left\{\|\sqrt{\delta} \mathcal{L} u\|_{K}^{2}+\|c u\|_{K}^{2}+\left\|u^{+}\right\|_{\partial_{-} K \cap \Gamma_{-}}^{2}\right. \\
& \left.+\frac{1}{2}\left\|u^{+}\right\|_{\partial_{+} K \cap \Gamma_{+}}^{2}+\frac{1}{2}\left\|u^{+}-u^{-}\right\|_{\partial_{-} K \backslash \Gamma_{-}}^{2}\right\} .
\end{aligned}
$$

Notice that for the SDFEM, the last term vanishes. Here is our main error estimate for the $h p$-DGFEM. 
Theorem 3.10 (Convergence rate of the hp-DGFEM)

Let $\Omega \subset \mathbb{R}^{2}$ and $\mathcal{T}, \mathcal{P}$ be as in Section 2 with (possibly irregular) mesh patches $\mathcal{T}_{P}, P \in \mathcal{P}$, consisting of shape-regular quadrilateral elements of degree $p_{K} \geq 1$. Select

$$
\left.\delta\right|_{K}=\delta_{K}=h_{K} / p_{K} \text { for all } K \in \mathcal{T}
$$

Then

$$
\left.\left\|u-u_{\mathrm{DG}}\right\|\right|_{\mathrm{DG}} ^{2} \leq C \sum_{K}\left(\frac{h_{K}}{2}\right)^{2 s_{K}+1} \frac{\Phi\left(p_{K}, s_{K}\right)}{p_{K}}|\hat{u}|_{s_{K}+1, \hat{K}}^{2},
$$

where $C>0$ depends only on elemental shape regularity, and the coefficients $a, b$, but is independent of $p_{K}, s_{K}, h_{K}$ and where $\Phi(p, s)$ is as in (3.11).

Proof Using (2.33) and Lemma 2.5 gives

$$
\begin{aligned}
\left\|u-u_{\mathrm{DG}}\right\| \|_{\mathrm{DG}} & \leq \quad\|\eta\|_{\mathrm{DG}}+\|\xi\|_{\mathrm{DG}} \\
\stackrel{(2.34)}{\leq} & \|\eta\|_{\mathrm{DG}}+\left(\sum_{K}\left\|\delta^{\frac{1}{2}} \mathcal{L} \eta-\delta^{-\frac{1}{2}} \eta\right\|_{K}^{2}\right)^{\frac{1}{2}} \\
& +2\left(\sum_{K}\|c \eta\|_{K}^{2}\right)^{\frac{1}{2}}+\sqrt{2}\left(\sum_{K}\left\|\eta^{+}\right\|_{\partial_{+} K \cap \Gamma_{+}}^{2}\right)^{\frac{1}{2}}+\left(\sum_{K}\left\|\eta^{-}\right\|_{\partial_{-} K \backslash \Gamma_{-}}^{2}\right)^{\frac{1}{2}} .
\end{aligned}
$$

Therefore,

$$
\begin{aligned}
\left\|u-u_{\mathrm{DG}}\right\| \|_{\mathrm{DG}} \leq & \left(\sum_{K}\left\|\delta^{\frac{1}{2}} \mathcal{L} \eta\right\|_{K}^{2}\right)^{\frac{1}{2}}+\left(\sum_{K}\|c \eta\|_{K}^{2}\right)^{\frac{1}{2}} \\
& +\left(\sum_{K}\left\|\eta^{+}\right\|_{\partial_{-} K \cap \Gamma_{-}}^{2}\right)^{\frac{1}{2}}+\frac{1}{\sqrt{2}}\left(\sum_{K}\left\|\eta^{+}\right\|_{\partial_{+} K \cap \Gamma_{+}}^{2}\right)^{\frac{1}{2}} \\
& +\frac{1}{\sqrt{2}}\left(\sum_{K}\left\|\eta^{+}-\eta^{-}\right\|_{\partial_{-} K \backslash \Gamma_{-}}^{2}\right)^{\frac{1}{2}}+\left(\sum_{K}\left\|\delta^{\frac{1}{2}} \mathcal{L} \eta\right\|_{K}^{2}\right)^{\frac{1}{2}}+\left(\sum_{K}\left\|\delta^{-\frac{1}{2}} \eta\right\|_{K}^{2}\right)^{\frac{1}{2}} \\
& +2\left(\sum_{K}\|c \eta\|_{K}^{2}\right)^{\frac{1}{2}}+\sqrt{2}\left(\sum_{K}\left\|\eta^{+}\right\|_{\partial_{+} K \cap \Gamma_{+}}^{2}\right)^{\frac{1}{2}}+\left(\sum_{K}\left\|\eta^{-}\right\|_{\partial_{-} K \backslash \Gamma_{-}}^{2}\right)^{\frac{1}{2}} \\
\leq & C\left\{\sum_{K}\left(\left\|\delta_{K}^{\frac{1}{2}} \nabla \eta\right\|_{K}^{2}+\left\|\delta_{K}^{\frac{1}{2}} \eta\right\|_{K}^{2}+\|\eta\|_{K}^{2}+\left\|\delta_{K}^{-\frac{1}{2}} \eta\right\|_{K}^{2}\right)\right. \\
& \left.+\sum_{K}\left(\left\|\eta^{+}\right\|_{\partial_{+} K \cap \Gamma_{+}}^{2}+\left\|\eta^{+}\right\|_{\partial_{-} K \cap \Gamma_{-}}^{2}+\left\|\eta^{-}\right\|_{\partial_{-} K \backslash \Gamma_{-}}^{2}+\left\|\eta^{+}\right\|_{\partial_{-} K \backslash \Gamma_{-}}^{2}\right)\right\}^{\frac{1}{2}} \\
\equiv & C(A+B)^{\frac{1}{2}},
\end{aligned}
$$

where $C$ depends on $(a, b)$.

We select $\eta=u-\Pi u$ with $\Pi$ as in Theorem 3.8. This gives the bound

$$
A \leq C \sum_{K}\left(\frac{h_{K}}{2}\right)^{2 s_{K}} \Phi\left(p_{K}, s_{K}\right)\left(\delta_{K}+\delta_{K}^{-1} h_{K}^{2} p_{K}^{-2}\right)|\hat{u}|_{s_{K}+1, \hat{K}}^{2} .
$$


To bound B, we must estimate $\|\eta\|_{\partial K}^{2}$. We use the inequality

$$
\|\eta\|_{\partial K}^{2} \leq C\left(\|\nabla \eta\|_{K}\|\eta\|_{K}+h_{K}^{-1}\|\eta\|_{K}^{2}\right) \quad \forall K \in \mathcal{T}
$$

and obtain the bound

$$
\begin{aligned}
B \leq & C \sum_{K}\left(\frac{h_{K}}{2}\right)^{s_{K}} \Phi\left(p_{K}, s_{K}\right)^{\frac{1}{2}}\left(\frac{h_{K}}{2}\right)^{s_{K}+1} \Phi\left(p_{K}, s_{K}\right)^{\frac{1}{2}} p_{K}^{-1}|\hat{u}|_{s_{K}+1, \hat{K}}^{2} \\
& +h_{K}^{-1}\left(\frac{h_{K}}{2}\right)^{2 s_{K}+2} \Phi\left(p_{K}, s_{K}\right) p_{K}^{-2}|\hat{u}|_{s_{K}+1, \hat{K}}^{2} \\
= & C \sum_{K}\left(\frac{h_{K}}{2}\right)^{2 s_{K}+1} p_{K}^{-1} \Phi\left(p_{K}, s_{K}\right)\left(1+p_{K}^{-1}\right)|\hat{u}|_{s_{K}+1, \hat{K}}^{2} .
\end{aligned}
$$

Selecting $\delta_{K}$ as in (3.20) concludes the proof.

An analogous error estimate holds true for the $h p$-SDFEM.

Theorem 3.11 (Convergence rate of the hp-SDFEM)

Let $\Omega \subset \mathbb{R}^{2}$ and $\mathcal{T}, P$ be as in Section 2 with a 1-irregular mesh consisting of shape-regular quadrilateral elements of degree $p_{K} \geq 1$. Select the stabilization parameter $\delta_{K}$ as in (3.20).

Then there holds the error estimate

$$
\left\|u-u_{\mathrm{SD}}\right\|_{\mathrm{SD}}^{2} \leq C \sum_{K}\left(\frac{h_{K}}{2}\right)^{2 s_{K}+1} \frac{\Phi\left(p_{K}, s_{K}\right)}{p_{K}}|\hat{u}|_{s_{K}+1, \hat{K}}^{2},
$$

where

$$
\|\| u\left\|_{\mathrm{SD}}^{2}:=\right\| \sqrt{\delta} \mathcal{L} u\left\|^{2}+\right\| c u\left\|^{2}+\frac{1}{2}\right\| u\left\|_{\Gamma_{+}}^{2}+\right\| u \|_{\Gamma_{-}}^{2}
$$

and

$$
0 \leq s_{K} \leq p_{K} \quad \forall K \in \mathcal{T}, \hat{u}=u \circ F_{P} \text { if } K \in \mathcal{T}_{P},
$$

and $\Phi(p, s)$ is as in (3.11).

The proof of Theorem 3.11 is completely analogous to that of Theorem 3.10, using Lemma 2.3 instead of Lemma 2.5.

Let us now discuss some special cases of the above, general error bounds.

\section{Remark 3.12}

1) If $p_{K}=p$ is fixed, and $h_{K}=h \rightarrow 0$, the bound (3.21) is optimal in $h$.

2) As $s$ is fixed and $p_{K}=p \rightarrow \infty$, Stirling's formula implies

$$
\Phi(p, s) \leq C(s) p^{-2 s}
$$

and (3.21) gives

$$
\left\|u-\left.u_{\mathrm{DG}}\left|\|_{\mathrm{DG}}^{2} \leq C \sum_{K}\left(\frac{h_{K}}{p_{K}}\right)^{2 s_{K}+1}\right| \hat{u}\right|_{s_{K}+1, \hat{K}} ^{2} .\right.
$$

The bound (3.21) is therefore optimal also in $p$. 
3) Suppose that $u$ is patch-wise analytic. Then,

$$
\forall \hat{K} \in \mathcal{T} \exists d_{K}>1, C>0 \quad \forall s>0:|\hat{u}|_{s, \hat{K}} \leq C\left(d_{K}\right)^{s} s !
$$

In this case, (3.21) gives exponential convergence, since picking $s=\alpha p$, with $0<\alpha<1$ to be selected below, and applying Stirling's formula gives

$$
\begin{aligned}
\Phi(p, s)|\hat{u}|_{s+1, \hat{K}}^{2} & \leq C\left(d_{K}\right)^{2 s+2}((s+1) !)^{2} \cdot \frac{(p-s) !}{(p+s) !} \\
& \leq C\left(d_{K}\right)^{2 \alpha p+2}(\alpha p+1)^{2 \alpha p+3} e^{-2 \alpha p-2} \frac{((1-\alpha) p)^{(1-\alpha) p} e^{-(1-\alpha) p}}{((1+\alpha) p)^{(1+\alpha) p} e^{-(1+\alpha) p}} \\
& \leq C p^{3}\left(F\left(\alpha, d_{K}\right)\right)^{p}
\end{aligned}
$$

where

$$
F(\alpha, d):=\frac{(1-\alpha)^{1-\alpha}}{(1+\alpha)^{1+\alpha}}(\alpha d)^{2 \alpha}
$$

Since, for $d>1$,

$$
\min _{0<\alpha<1} F(d, \alpha)=F\left(d, \alpha_{\min }\right)<1, \quad \alpha_{\min }=\frac{1}{\sqrt{1+d^{2}}}
$$

it follows, setting $2 b_{K}=\left|\log F\left(d_{K}, \alpha_{\text {min }}\right)\right|$, that

$$
\Phi\left(p_{K}, \alpha p_{K}\right)|\hat{u}|_{\alpha p_{K}+1, \hat{K}}^{2} \leq C p_{K}^{3} e^{-2 b_{K} p_{K}},
$$

and we get from (3.21) the exponential convergence estimate

$$
\left\|u-u_{\mathrm{DG}}\right\|_{\mathrm{DG}}^{2} \leq C \sum_{K}\left(\frac{h_{K}}{2}\right)^{2 s_{K}+1} p_{K}^{2} e^{-2 b_{K} p_{K}} .
$$

By Theorem 3.11 an analogous bound holds also for the $h p$-SDFEM on quadrilateral, possibly 1-irregular meshes.

Finally, we note that exponential convergence estimates analogous to the ones presented here on quadrilaterals can also be proved on triangular meshes, using the approximation results of Braess and Schwab [2]. Further aspects of the local discontinuous Galerkin method will be considered in [3].

\section{Numerical experiments}

In this section we present a number of numerical experiments to verify the a priori error estimates derived in Section 3.4 for both the $h p$-DGFEM and the $h p$-SDFEM. 


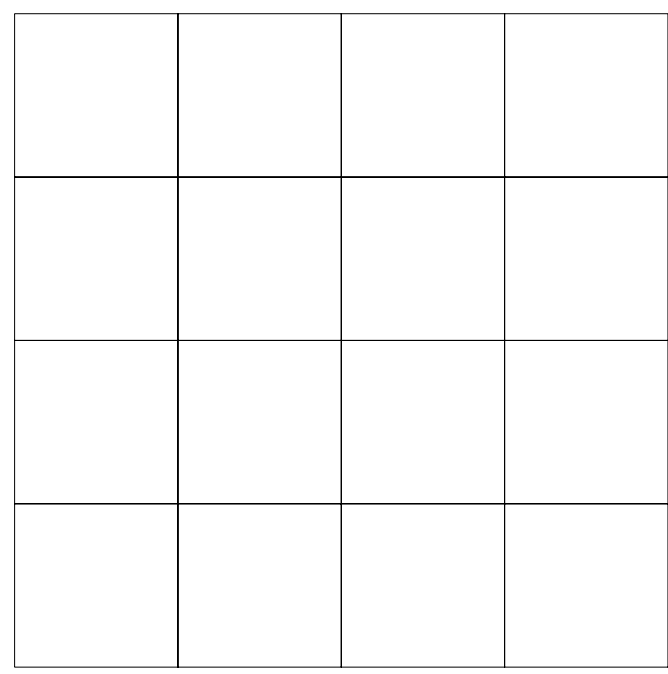

(a)

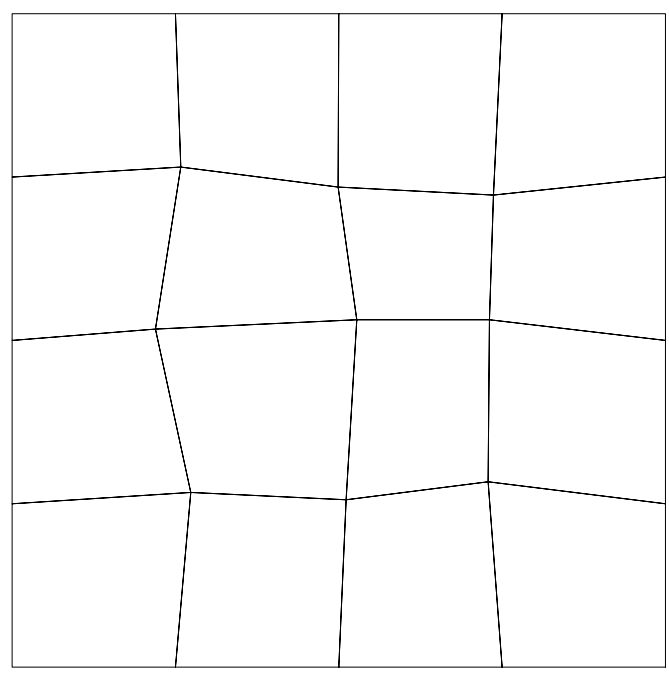

(b)

Figure 5: Example 1. (a) Uniform $5 \times 5$ square mesh; (b) Quadrilateral mesh based on a $10 \%$ random perturbation of mesh (a).

\subsection{Example 1}

In this example we let $\Omega=(-1,1)^{2}, a=(8 / 10,6 / 10), b=1, g=1$ and $f$ is chosen so that the analytical solution to $(2.1)$ is given by

$$
u(x, y)=1+\sin \left(\pi(1+x)(1+y)^{2} / 8\right)
$$

cf. [1].

We first investigate the asymptotic behaviour of the $h p$-DGFEM on a sequence of successively finer square and quadrilateral meshes for different $p$. In each case, the quadrilateral mesh is constructed from a uniform $N \times N$ square mesh by randomly perturbing each of the interior nodes by up to $10 \%$ of the local mesh size: Figure 5 shows an example of a $5 \times 5$ square mesh together with the corresponding quadrilateral mesh.

In Figure 6 we first present a comparison of the DG-norm of the error with the mesh function $h$ for $p$ ranging between 1 and 5 . Here, we clearly see that $\left\|u-u_{\mathrm{DG}}\right\|_{\text {DG }}$ converges like $\mathcal{O}\left(h^{p+1 / 2}\right)$ as $h$ tends to zero for each (fixed) $p$. Secondly, we investigate the convergence of the DGFEM with $p$-enrichment for fixed $h$. Since the true solution (4.1) is a real analytic function, we expect to observe exponential rates of convergence, cf. Remark 3.12. Indeed, Figure 7 clearly illustrates this behaviour: on the linear-log scale, the convergence plots for each $p$ become straight lines as the degree of the approximating polynomial is increased. Furthermore, we observe from Figures $6 \& 7$ that the $h$-and $p$-convergence, respectively, of the DGFEM is robust with respect to mesh distortion.

Finally, we verify the a priori error bound (3.22) for the $h p$-SDFEM. In Figures $8 \& 9$ we show the convergence of the scheme with respect to both $h-$ and $p$-refinement, respectively. As with the DGFEM, we again observe optimal rates of convergence as $h$ tends to zero for fixed $p$ (Figure 8) and exponential rates of convergence for fixed $h$ as $p$ is increased (Figure 


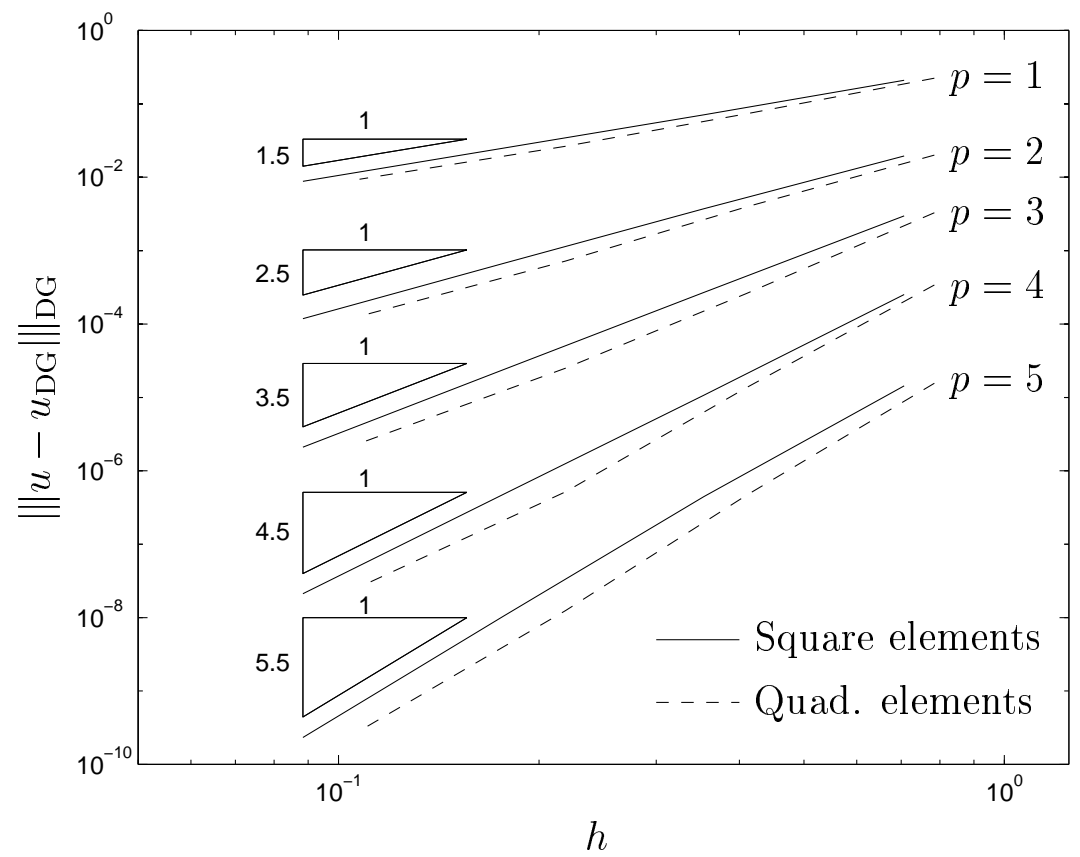

Figure 6: Example 1. Convergence of the DGFEM with $h$-refinement.

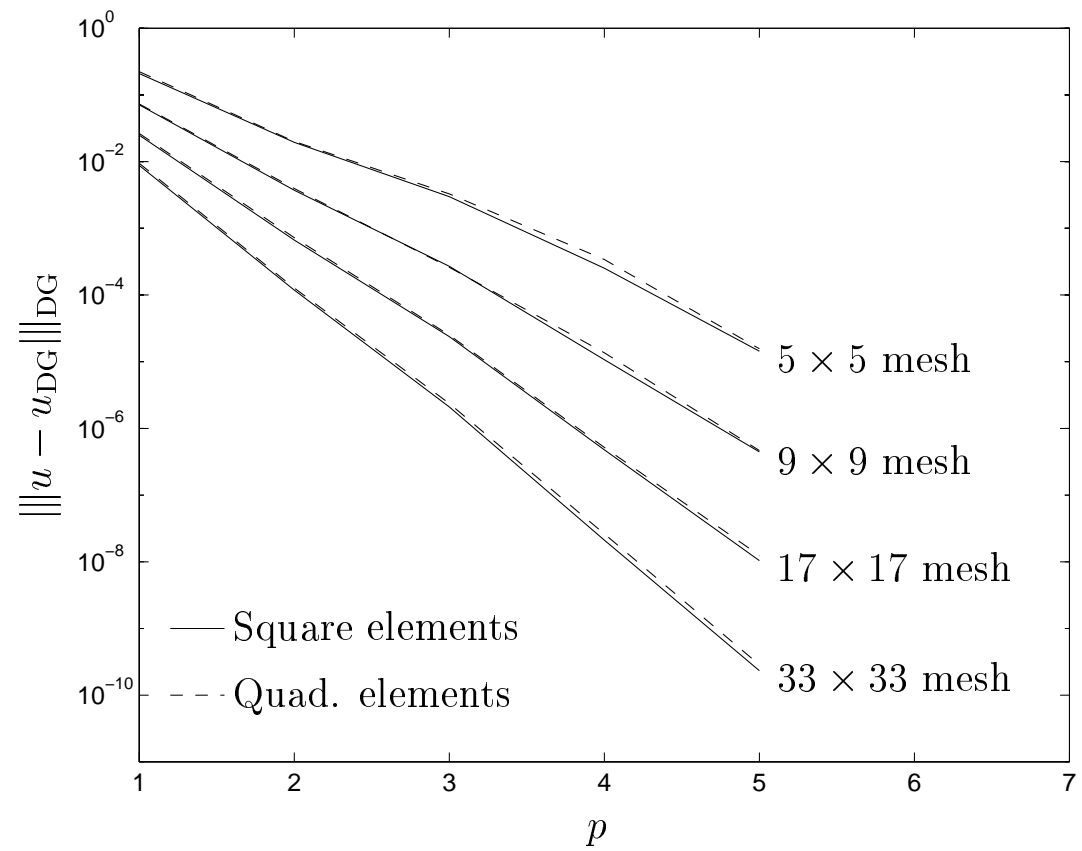

Figure 7: Example 1. Convergence of the DGFEM with $p$-refinement. 


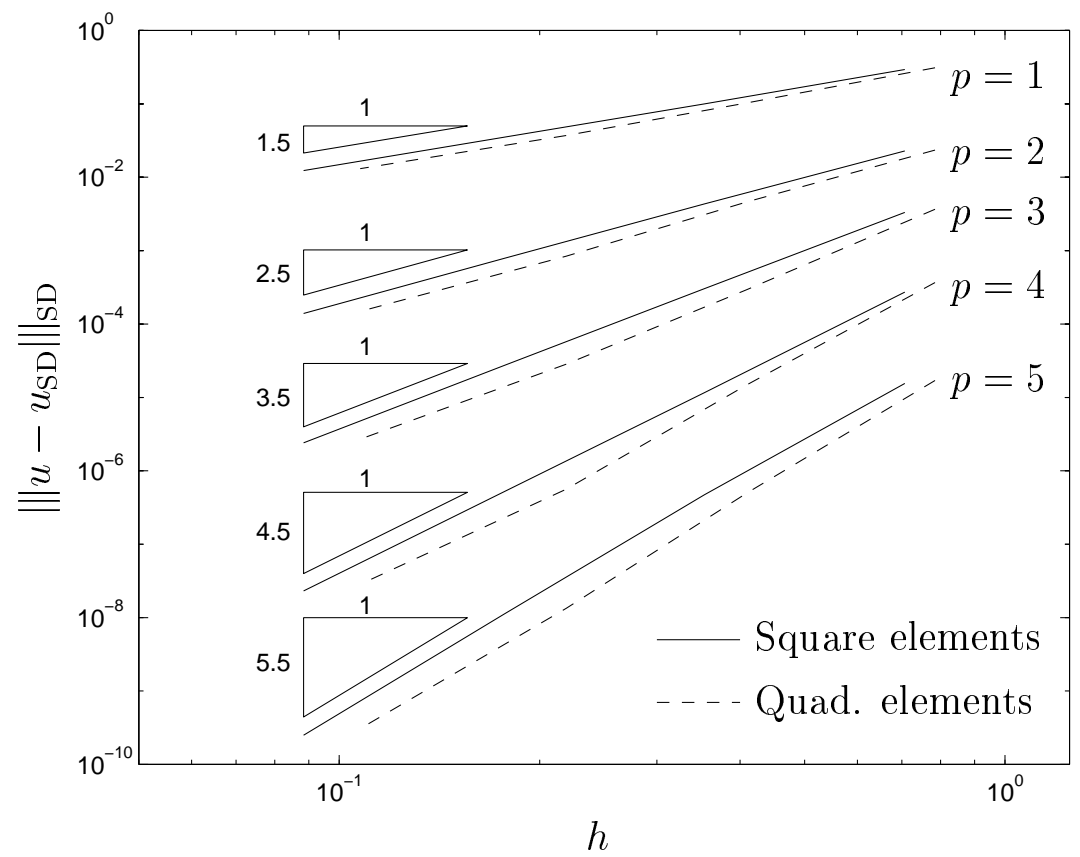

Figure 8: Example 1. Convergence of the SDFEM with $h$-refinement.

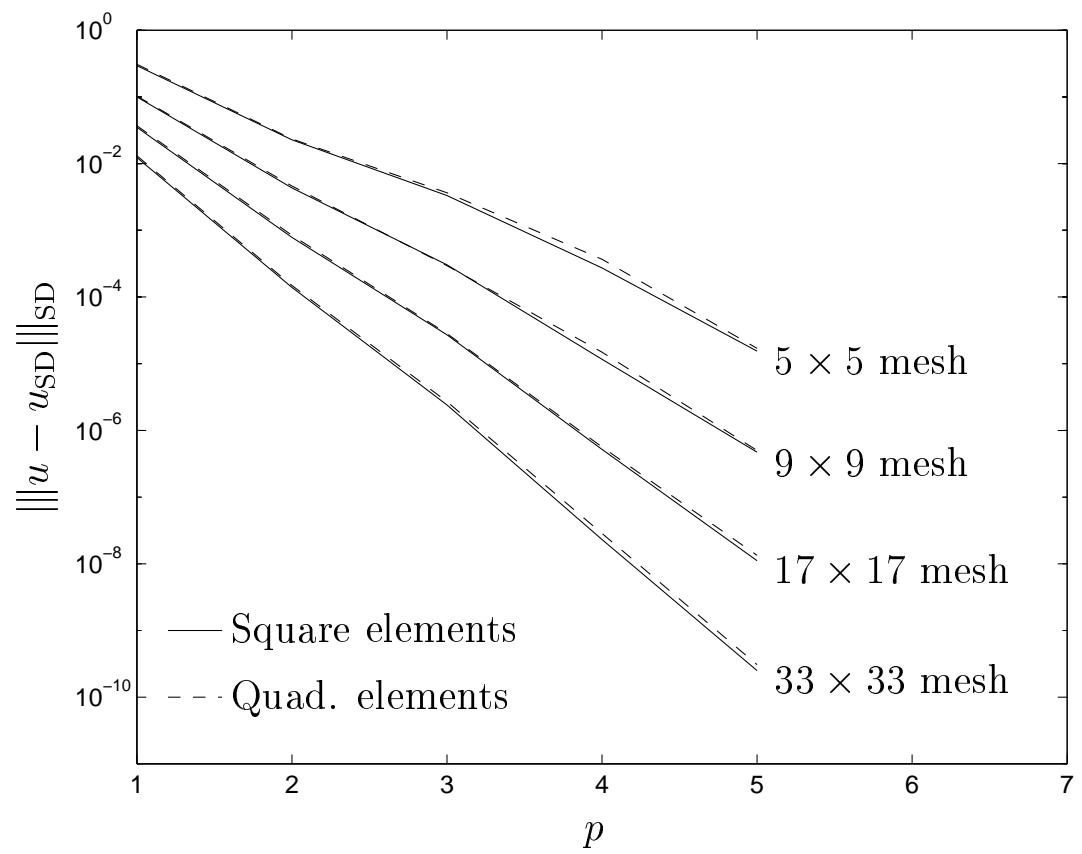

Figure 9: Example 1. Convergence of the SDFEM with $p$-refinement. 


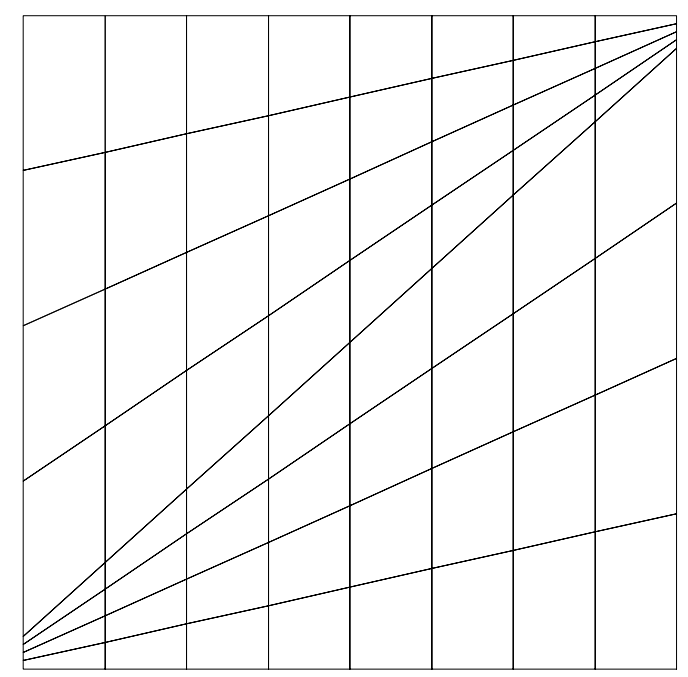

Figure 10: Example 2. $9 \times 9$ quadrilateral mesh aligned with the discontinuity.

9) on both uniform square meshes and quasi-uniform quadrilateral meshes. We remark that in all the computations performed here, the DGFEM was marginally more accurate than the SDFEM for each $h$ and $p$; though, of course, the number of degrees of freedom in the DGFEM is greater than in the SDFEM for a given $h$ and $p$.

\subsection{Example 2}

In this example we let $\Omega=(-1,1)^{2}, a=(1,9 / 10), b=1$ and $f$ is chosen so that the analytical solution to (2.1) is given by

$u(x, y)=\left\{\begin{array}{cl}\sin \left(\pi(x+1)^{2} / 4\right) \sin (\pi(y-9 x / 10) / 2) & \text { for }-1 \leq x \leq 1,9 x / 10<y \leq 1 \\ \mathrm{e}^{-5\left(x^{2}+(y-9 x / 10)^{2}\right)} & \text { for }-1 \leq x \leq 1, \quad-1 \leq y<9 x / 10\end{array}\right.$

thus, $u$ is discontinuous along the line $y=9 x / 10$.

To demonstrate the advantage of using discontinuous elements, we now only consider $N \times N$ quadrilateral meshes which are aligned with the discontinuity; choosing $N$ to be odd ensures that the discontinuity lies on element interfaces, cf. Figure 10. In this case the DGFEM does not 'see' the lack of regularity in the problem and behaves as if the analytical solution $u$ were smooth; i.e. optimal algebraic rates of convergence are observed with $h-$ refinement and exponential rates of convergence are observed with $p$-refinement. These results are summarized in Figure 11, where we show $\left|\left\|u-u_{\mathrm{DG}} \mid\right\|_{\mathrm{DG}}\right.$ in terms of the number of degrees of freedom. Thus, in practice, if an adaptive refinement strategy is implemented which is capable of aligning the mesh with localised structures in the solution such as shocks, cf. [6] for example, then optimal, and indeed exponential, rates of convergence will be attained with the DGFEM. In contrast, from Figure 12 we observe that the convergence rate of the SDFEM is limited by the regularity of $u$; we remark that by aligning the mesh with the discontinuity improves the accuracy of the SDFEM, though the rate of convergence of the scheme with $h$ - and $p$-refinement is not enhanced. 


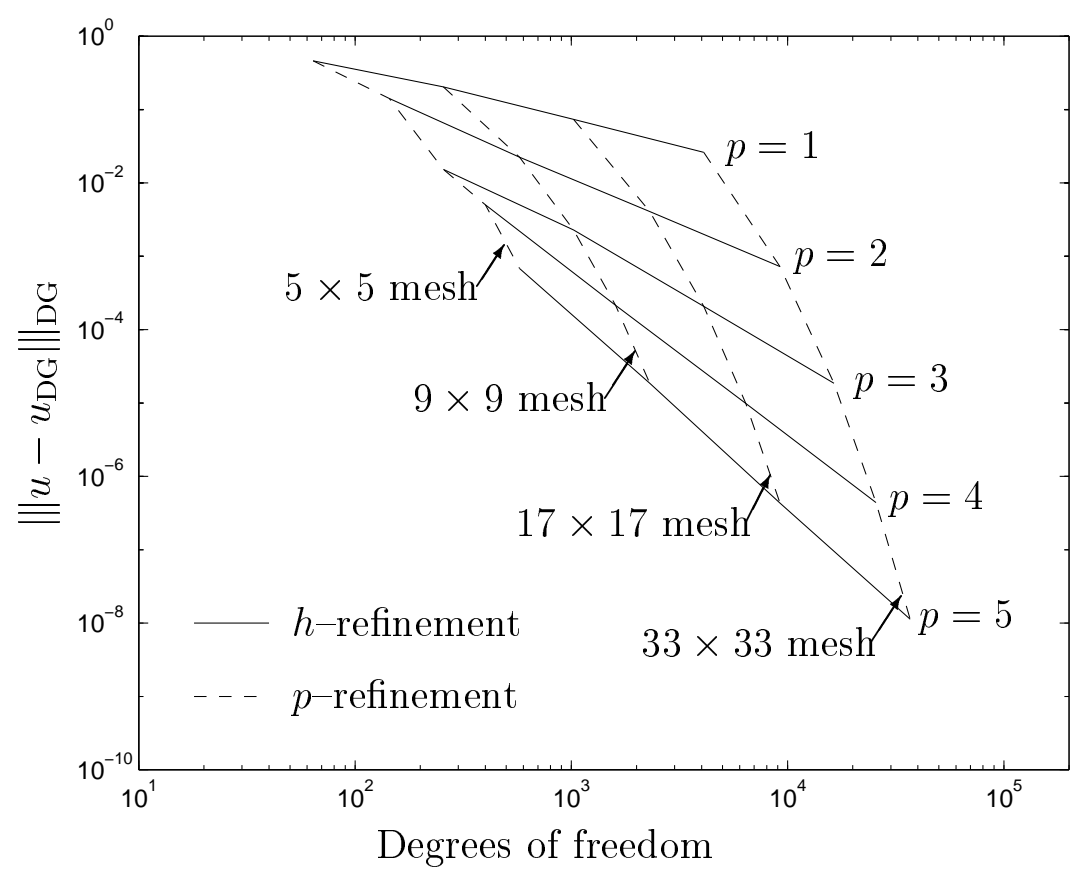

Figure 11: Example 2. Convergence of the DGFEM with $h p$-refinement on quadrilateral meshes aligned with the discontinuity.

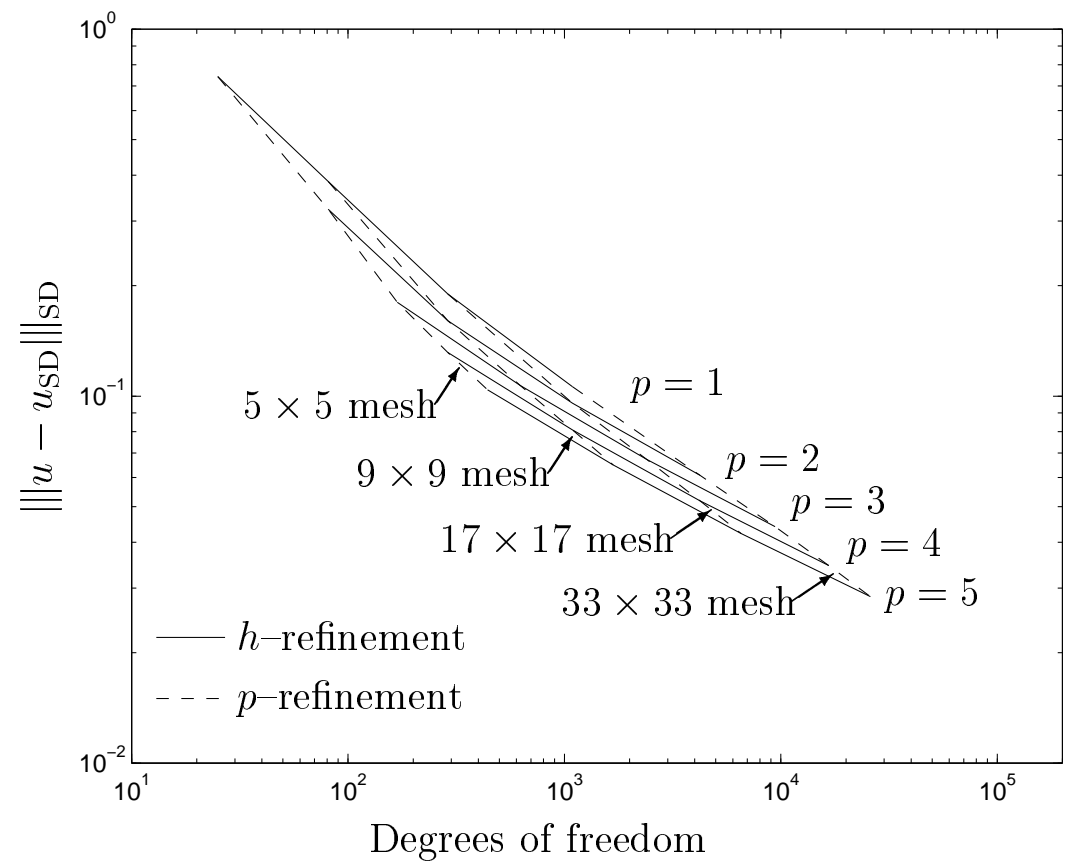

Figure 12: Example 2. Convergence of the SDFEM with $h p$-refinement on quadrilateral meshes aligned with the discontinuity. 
Finally, we note that if the mesh is not aligned with the discontinuity, then the DGFEM convergences at the same (slow) rate as the SDFEM; though, in all the numerical computations performed here, the DGFEM was marginally more accurate than the SDFEM for each $h$ and $p$, cf. Example 1.

\section{References}

[1] K.S. Bey and J.T. Oden, hp-Version discontinuous Galerkin methods for hyperbolic conservation laws. Comput. Methods Appl. Mech. Engrg. 133 (1996), pp. 259-286.

[2] D. Braess and C. Schwab, Approximation on triangles with respect to weighted Sobolev norms. (In preparation).

[3] B. Cockburn and C. Schwab, hp-error analysis for the local discontinuous Galerkin method. (In preparation).

[4] B. Cockburn, S. Hou, and C.-W. Shu, TVB Runge-Kutta local projection discontinuous Galerkin finite elements for hyperbolic conservation laws. Math. Comp. 54 (1990), pp. 545-581.

[5] L. Demkowicz, K. Gerdes, C. Schwab, A. Bajer, and T. Walsh. HP90: A general and flexible Fortran $90 \mathrm{hp}$-FE code. HP90. Report 97-17, SAM, ETH-Zürich. Computing and Visualization in Science. (To appear). Available from the URL ftp://ftp.sam.math.ethz.ch/pub/sam-reports/reports/reports97/97-17.ps.Z

[6] P. Houston, J. Mackenzie, E. Süli, and G. Warnecke, A posteriori error analysis for numerical approximations of Friedrichs systems. Numerische Mathematik. (To appear). Available from the URL http://www.comlab.ox.ac.uk/oucl/users/paul.houston/ friedrichs.ps.gz

[7] C. Johnson, U. Nävert, and J. Pitkäranta, Finite Element Methods for linear hyperbolic problems. Comp. Meth. Appl. Mech. Engrg. 45 (1984) pp. 285-312.

[8] C. Johnson and J. Pitkäranta, An analysis of the discontinuous Galerkin method for a scalar hyperbolic conservation law. Math. Comp. 46 (1986) pp. 1-23.

[9] P. Lesaint and P.A. Raviart, On a finite element method for solving the neutron transport equation. In: Mathematical aspects of Finite Elements in Partial Differential Equations, C.A. deBoor (Ed.), Academic Press New York (1974), pp. 89-123.

[10] C. Schwab, $p$ - and hp-Finite Element Methods. Theory and Applications to Solid and Fluid Mechanics. Oxford University Press, 1998. 